\title{
THERMAL RADIATION AND CHEMICAL REACTION EFFECTS ON UNSTEADY MAGNETOHYDRODYNAMIC THIRD GRADE FLUID FLOW BETWEEN STATIONARY AND OSCILLATING PLATES
}

\author{
A.S. IDOWU* and U. SANI \\ Department of Mathematics, University of Ilorin \\ Kwara State, NIGERIA \\ E-mail: asidowu@gmail.com
}

\begin{abstract}
An analysis was carried out for an unsteady magnetohydrodynamic(MHD) flow of a generalized third grade fluid between two parallel plates. The fluid flow is a result of the plate oscillating, moving and pressure gradient. Three flow problems were investigated, namely: Couette, Poiseuille and Couette-Poiseuille flows and a number of nonlinear partial differential equations were obtained which were solved using the He-Laplace method. Expressions for the velocity field, temperature and concentration fields were given for each case and finally, effects of physical parameters on the fluid motion, temperature and concentration were plotted and discussed. It is found that an increase in the thermal radiation parameter increases the temperature of the fluid and hence reduces the viscosity of the fluid while the concentration of the fluid reduces as the chemical reaction parameter increases.
\end{abstract}

Key words: third grade fluid, plane Couette, Poiseuille and Couette-Poiseuille flow, magnetohydrodynamics flow, stationary and oscillating plate, thermal radiation and chemical reaction.

\section{Introduction}

A fluid is a substance which is capable of flowing or on the other hand we can say that a fluid is a substance which deforms continously when subjected to an external shearing force. Fluids may be of the following types: Newtonian, Non-Newtonian, plastic and ideal. Thus, considering Non-Newtonian fluids, which are fluids that do not obey the Newton law of viscosity and the relation between shear stress and rate of shear strain is nonlinear. Non-Newtonian fluids are increasingly considered as more important and appropriate in technological applications in several industrial manufacturing processes such as in the drilling of oil and gas wells, polymer and petroleum industries, power generators, pumps as well as accelerators.

A large class of real fluids does not exibit a linear relationship between stress and the rate of strain, so because of their nonlinear dependence, the analysis of the behaviour of fluid motion of non-Newtonian fluids tends to be more complicated in comparison with that of a Newtonian fluid. Due to the complexity of these fluids several constitutive equations have been proposed which are complicated and contain some special cases of these fluids. The constitutive equations of viscoelastic fluids are usually classified under the categories of differential, rate and integral models. The fluid under consideration falls under the category of the differential model with a very short history of the deformation gradient which has an influence on the stress. However, there is no model which can alone predict the behavior of all non-Newtonian fluids and governing equation due to the complexity of these fluids.

In view of this type of fluid and its applications, many researchers have studied steady and unsteady magnetohydrodynamic(MHD) third grade fluids flow between parallel plates with different configurations. Hayat et al. [1] developed a set of differential equations describing the steady flow of an Oldroyd 6-constant magnetohydrodynamic fluid and found that the obtained solutions for the steady flow are strongly dependent on the material constant (non-Newtonian parameter) and it differs from the model of Oldroyd 3-constant

\footnotetext{
* To whom correspondence should be addressed
} 
fluid. Closed form solutions for the velocity and temperature profiles were obtained for a particular viscoelastic fluid between two oscillatory walls in the presence of transverse magnetic field by Ajadi [2], where the flow is due to the continous movement of the oscillatory wall and the time dependent pressure gradient and there is a monotonic decrease in velocity with time in the domain while the viscoelastic fluid velocity decreases as the distance from the wall decreases while it is on the contrary for the non-viscoelastic fluid $(E \neq 0)$. Aksoy and Pakdemili [3] investigated the flow of a non-Newtonian fluid through porous media between two parallel plates at different temperatures. The governing momentum equation of a third grade fluid with modified Darcy's law and energy equation were derived. The approximate analytical solutions obtained by using perturbation techniques were within the validity range, the analytical and numerical solutions are same. The heat transfer analysis on the laminar flow of an incompressible third grade fluid through a porous flat channel was studied by Ellahi et al. [4] where the lower plate was assumed to be at a higher temperature than the upper plate. The obtained analytical solutions were compared with the numerical solutions and showed that accuracy is remarkable. Hayat and Nawaz [5] investigated the effects of heat transfer on a magnetohydrodynamic(MHD) axisymetric flow of a viscous fluid between two radially stretching sheets and the solutions presented by using the homotopy analysis method show that the magnetic field slows down the motion of the fluid while the dimensionless radial velocity decreases when the porosity parameter increases. Danish et al. [6] obtained the exact analytical solutions for the velocity profiles and flow rates in an explicit form for the Poiseuille and Couette-Poiseuille flow of a third grade fluid flow between two parallel plates. The exact solutions match well with their numerical counterpart and better than the recently developed approximate analytical solutions. Radiation and heat transfer effects on an MHD nonNewtonian unsteady flow in a porous medium with slip condition were investigated by Gbadeyan and Dada [7] where the fluid is assumed not to absorb its own emitted radiation but that of the boundaries. Crank Nicolson type of the finite difference method was used to solve the resulting governing equations.

An unsteady MHD thin film of a third grade fluid down an inclined plane with no slip boundary condition was studied by Aiyesimi et al. [8] and it was found that the magnetic field decreases the viscous and Joule dissipation, while it decreases the velocity profile and its gradient. Aiyesimi et al. [9] investigated the combined effects of the magnetic field on the MHD flow of a third grade fluid through an inclined channel in the presence of a uniform magnetic field with the consideration of heat transfer. Also, three different problems, i.e, Couette, Poiseuille and Couette-Poiseuille flows, were analysed and it was observed that the velocity increases rapidly from the moving upper plate to the stationary lower plate and the velocity of the fluid increases through the two stationary plates as the gravitational parameter increases. Baoku [10] presented the effects of suction and thermal radiation on the unsteady convective flow and heat transfer in a third grade fluid flow over an infinite vertical plate where the plate is porous to allow possible wall suction. The Crank-Nicolson finite difference scheme was empolyed to solve the governing time-based coupled partial differential equations where the flow field is appreciably influenced by suction and viscoelastic parameters. Gul et al. [11] studied the thin film flow in an MHD third grade fluid on a vertical belt with temperature dependent viscosity by using the Adomian decomposition method to solve the governing coupled nonlinear differential equations with their boundary conditions. Hayat et al. [12] addressed the boundary layer flow and heat transfer in a third grade fluid over an unsteady permeable stretching sheet. The transverse magnetic and electric fields in the momentum equation are considered. It is observed that both the velocity and temperature profiles increase in the presence of an electric field while the temperature increases due to the radiation parameter. The boundary layer thickness increases by increasing the Eckert number. Rasheed et al. [13] considered an unsteady magnetohydrodynamic flow of a generalized third grade fluid between two parallel plates. The flow is caused by the plate oscillation and movement. The solutions to the nonlinear partial differential equations are obtained by using the homotopy perturbation method. It was observed that the velocity field decreases by increasing the magnetic term. Shah et al. [14] worked on the flow of an unsteady non-Newtonian fluid between stationary and oscillating plates and solved the nonlinear partial differential equations using the homotopy perturbation method. It was observed that the velocity increases by increasing the pressure gradient parameter. Hayat et al. [15] examined the effects of an inclined magnetic field and heat transfer in the flow of a third grade fluid with an exponentially stretching surface. The resulting equations are solved for the approximate solutions and effects of fluid parameters on the 
temperature field are quite similar for heat generation and absorption. Makinde et al. [16] made a thermodynamic analysis of variable viscosity hydromagnetic Couette flow in a rotating system with Hall effects and showed that the fluid rotation increases the dominant effect of heat transfer irreversibility at the upper moving plate region while the entropy production is more at the lower fixed plate region. An unsteady MHD flow and heat transfer of a third grade fluid past on oscillating inclined belt was studied by Nasir et al. [17] where the analytical solutions of velocity and temperature profiles were obtained by using the optimal homotopy asymptotic method and homotopy perturbation method. Ghani et al. [18] examined the motion of an incompressible unidirectional magnetohydrodynamics thin film flow of a third grade fluid over an oscillating inclined belt embedded in a porous medium and the physical problem was modeled in terms of nonlinear partial differential equations solved using two analytical techniques, namely the optimal homotopy asymptotic method and homotopy perturbation method. An unsteady two-dimensional MHD flow between the infinite parallel plate was investigated by Kumar et al. [19] and the effect of the thermal radiation and magnetic field were included in this model. The transformed governing equation was solved numerically by using the Runge-Kutta shooting technique and it was noticed that the Nusselt number is an increasing function of the squeeze number. However, based on these works, it has been observed that the effects of thermal radiation and chemical reaction on an unsteady MHD fluid flow between stationary and oscillating plates have not been considered.

\section{Mathematical formulation of the problem}

An incompresible simple fluid is defined as a material whose state of present stress is determined by the history of the deformation gradient without a preferred reference configuration. As such the fluid flow problem is governed by the continuity, momentum and temperature equations, respectively

$$
\nabla \cdot \boldsymbol{V}=0
$$

where $\boldsymbol{V}=(u, v, w)$ is the velocity vector

The velocity field $\boldsymbol{V}$ for the fluid flow problem under investigation should be of the form

$$
\boldsymbol{V}=[u(y, t), 0,0]
$$

where $u(y, t)$ is the velocity field in the $x$-direction. This velocity field satisfies the continuity Eq.(2.1).

The relevant momentum equation is given by

$$
\rho \frac{\partial u}{\partial t}=\mu \frac{\partial^{2} u}{\partial y^{2}}+\alpha_{1}\left(\frac{\partial^{3} u}{\partial y^{2} \partial t}\right)+6 \beta_{3}\left(\frac{\partial u}{\partial y}\right)^{2} \frac{\partial^{2} u}{\partial y^{2}}-\sigma B_{0}^{2} u .
$$

From the Maxwell equation which has the form

$$
\rho C_{p} \frac{D \theta}{D t}=k \nabla^{2} \theta+\operatorname{tr}(\boldsymbol{T} \cdot L)-\frac{\partial q_{r}}{\partial y}
$$

where $\rho$ is the constant fluid density, $\boldsymbol{T}$ is the stress tensor, $C_{p}$ is the specific heat, $k$ is the thermal conductivity, $\theta$ is the temperature, $\frac{D}{D t}$ is the material derivative, $L=\operatorname{grad} V, q$ is the radiative heat flux according to Rosseland approximation where

$$
q_{r}=\frac{-16 \sigma^{*} \boldsymbol{T}^{3}}{3 k^{*}} \frac{\partial \boldsymbol{T}}{\partial y}
$$


where $\sigma^{*}$ is the Stefan-Boltzman constant, $k^{*}$ is the mean absorption coefficient. Note that in writing Maxwell's equation the displacement current is neglected.

And also the equation for concentration governing the flow is given by

$$
\frac{\partial C}{\partial t}+u \frac{\partial C}{\partial x}+v \frac{\partial C}{\partial y}+w \frac{\partial C}{\partial z}=D \frac{\partial^{2} C}{\partial y^{2}}-K_{r} C
$$

where $u, v$ are the velocities in the $x, y$ directions, respectively, $C$ is the concentration, $D$ is the diffusion coefficient of the diffusing species and $K r$ the chemical reaction parameter.

\section{Formulation of the plane Couette flow problem}

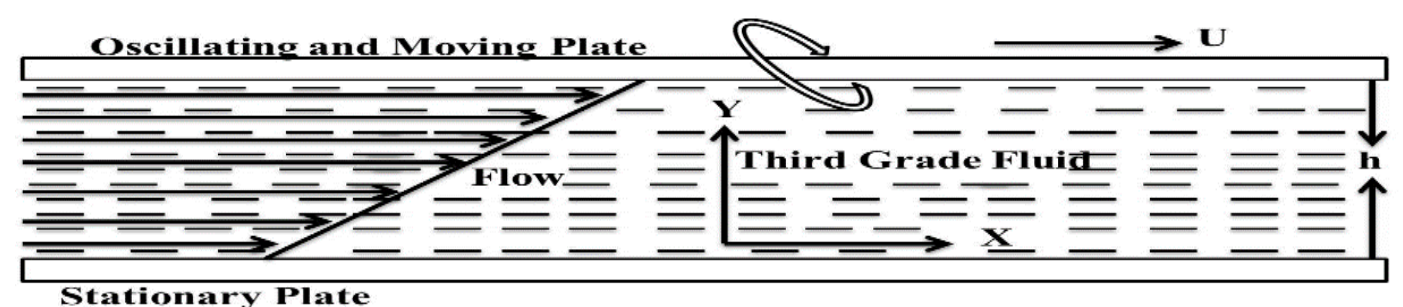

Fig.1. Model of the plane Couette flow.

Consider an unsteady laminar flow of an incompressible third grade fluid between two parallel plates. The upper plate is oscillating and moving with constant velocity $U$ relative to the lower plate. The upper plate carries along with itself a liquid of a third grade fluid during its motion. The upper and lower plates have a uniform distance between them considered to be $h$. The temperature of the higher and the lower plate are $\theta_{1}$ and $\theta_{0}$, considering MHD unidirectional flow with zero pressure gradient with a uniform applied magnetic field $B_{0}$ acting in the y-direction. Thus having $\boldsymbol{V}[u(y, t), 0,0], \theta(y, t)$ and $C(y, t)$, also the above definition of velocity satisfies the continuity equation. The momentum, energy and concentration equations yield

$$
\begin{aligned}
& \rho \frac{\partial u}{\partial t}=\mu\left(\frac{\partial^{2} u}{\partial y^{2}}\right)+\alpha_{1}\left(\frac{\partial^{3} u}{\partial y^{2} \partial t}\right)+6 \beta_{3}\left(\frac{\partial u}{\partial y}\right)^{2} \frac{\partial^{2} u}{\partial y^{2}}-\sigma B_{0}^{2} u \\
& \rho C_{p} \frac{\partial \theta}{\partial t}=k\left(\frac{\partial^{2} \theta}{\partial y^{2}}\right)+\mu\left(\frac{\partial u}{\partial y}\right)^{2}+\alpha_{1} \frac{\partial^{3} u}{\partial y^{2} \partial t}+2 \beta_{3}\left(\frac{\partial u}{\partial y}\right)^{4}+\frac{16 \sigma^{*} \theta_{0}^{3}}{3 k^{*}} \frac{\partial^{2} \theta}{\partial y^{2}} \\
& \frac{\partial C}{\partial t}=D\left(\frac{\partial^{2} C}{\partial y^{2}}\right)-K_{r} C
\end{aligned}
$$

with intial and boundary conditions

$$
\begin{aligned}
& u(h, t)=U+U \cos (\omega h), \quad(y>0), \quad u(0, t)=U+U \cos (\omega t), \quad(t>0), \\
& u(y, t)=0, \quad \text { at } y \rightarrow \infty(t>0),
\end{aligned}
$$




$$
\begin{aligned}
& \theta(h, t)=\left(\theta_{1}-\theta_{0}\right)+\left(\theta_{1}-\theta_{0}\right) \cos (\omega h)+\theta_{0}, \quad(y>0), \\
& \theta(0, t)=\left(\theta_{1}-\theta_{0}\right)+\left(\theta_{1}-\theta_{0}\right) \cos (\omega t)+\theta_{0}, \quad(t>0), \\
& C(h, t)=\left(C_{1}-C_{0}\right)+\left(C_{1}-C_{0}\right) \cos (\omega y)+C_{0}, \quad(y>0), \\
& C(0, t)=\left(C_{1}-C_{0}\right)+\left(C_{1}-C_{0}\right) \cos (\omega t)+C_{0}, \quad(t>0) .
\end{aligned}
$$

Here $\omega$ is the frequency of the oscillating plate. We introduce the non-dimensionless quantities

$$
\bar{u}=\frac{u}{U}, \quad \bar{y}=\frac{y}{h}, \quad \bar{t}=\frac{t \mu}{\delta^{2} \rho}, \quad \bar{\theta}=\frac{\theta-\theta_{0}}{\theta_{1}-\theta_{0}}, \quad \bar{C}=\frac{C-C_{0}}{C_{1}-C_{0}},
$$

applying Eqs (3.7) to Eqs (3.1), (3.2), (3.3), (3.4), (3.5) and (3.6), where for simplicity we drop the bars of the dimensionless quantities yields

$$
\begin{aligned}
& \frac{\partial u}{\partial t}=\delta_{*}\left(\frac{\partial^{2} u}{\partial y^{2}}\right)+\alpha\left(\frac{\partial^{3} u}{\partial y^{2} \partial t}\right)+6 \beta\left(\frac{\partial u}{\partial y}\right)^{2}\left(\frac{\partial^{2} u}{\partial y^{2}}\right)-M u \\
& \frac{\partial \theta}{\partial t}=\frac{\delta_{*}}{\operatorname{Pr}}\left(\frac{\partial^{2} \theta}{\partial y^{2}}\right)+\delta_{*} \operatorname{Ec}\left(\frac{\partial u}{\partial y}\right)^{2}+\alpha \operatorname{Ec} \frac{\partial^{3} u}{\partial y^{2} \partial t}+\beta \operatorname{Ec}\left(\frac{\partial u}{\partial y}\right)^{4}+\frac{R}{\operatorname{Pr}} \frac{\partial^{2} \theta}{\partial y^{2}} \\
& \frac{\partial C}{\partial t}=\frac{\delta_{*}}{\operatorname{Sc}}\left(\frac{\partial^{2} C}{\partial y^{2}}\right)-\frac{K r_{*}}{\operatorname{Sc}}(C+N c) \\
& u(y, 0)=1+\cos (\omega y), \quad u(0, t)=1+\cos (\omega t), \quad u(\infty, t)=0 \\
& \theta(y, 0)=1+\cos (\omega y), \quad \theta(0, t)=1+\cos (\omega t), \\
& C(y, 0)=1+\cos (\omega y), \quad C(0, t)=1+\cos (\omega t)
\end{aligned}
$$

where

$$
\begin{aligned}
& \alpha=\frac{\alpha_{1}}{\rho h^{2}}, \quad \beta=\frac{\beta_{3} U^{2} \delta^{2}}{\mu h^{4}}, \quad M=\frac{\sigma B_{0}^{2} \delta^{2}}{\mu}, \quad \delta_{*}=\frac{\delta^{2}}{h^{2}}, \quad \mathrm{Ec}=\frac{U^{2}}{C p\left(\theta_{1}-\theta_{0}\right)}, \\
& \frac{1}{\operatorname{Pr}}=\frac{k}{\mu C p}, \quad R=\frac{\sigma^{2} \delta^{2} \theta_{0}^{3}}{h^{2} k^{*} k}, \quad \frac{1}{\mathrm{Sc}}=\frac{\rho D}{\mu}, \quad K r_{*}=\frac{K r \delta^{2}}{D}, \quad N c=\frac{C_{0}}{\left(C_{1}-C_{0}\right)} .
\end{aligned}
$$

The parameters above in Eq.(3.14) encompass the physics of the problem. In particular, $\alpha$ and $\beta$ are the non-Newtonian parameters, $\delta_{*}$ is a constant parameter, Ec is the Eckert number, $M$ is the magnetic field parameter, $\mathrm{Pr}$ is the Prandtl number, $R$ is the radiation parameter, $\mathrm{Sc}$ is the Schmidt number, $K r_{*}$ is the chemical reaction parameter, $N c$ is the concentration difference parameter. 


\section{Method of solution}

We employed the method of solution called the He-Laplace method by Hradyesh and Atulya [20], where the use of the homotopy pertubation method coupled with the Laplace transformation method to solve linear and nonlinear partial differential equations was employed. It is worth mentioning that the method is an elegant combination of the Laplace transformation, the homotopy perturbation method and He's polynomials. The use of He's polynomials in the nonlinear term was first introduced by Ghorbani and Saberi-Nadjafi [21] and Ghorbani [22]. To illustrate the basic idea of this method, let us consider a general nonlinear nonhomogeneous partial differential equation with initial conditions of the form

$$
\begin{aligned}
& \frac{\partial^{2} y}{\partial t^{2}}+R_{1} y(x, t)+R_{2} y(x, t)+N y(x, t)=f(x, t), \\
& y(x, 0)=\alpha(x), \quad \frac{\partial y}{\partial t}(x, 0)=\beta(x)
\end{aligned}
$$

where $R_{1}=\frac{\partial^{2}}{\partial x^{2}}$ and $R_{2}=\frac{\partial}{\partial x}$ are the linear differential operators, $N$ represents the general nonlinear differential operator and $f(x, t)$ is the source term. Taking the Laplace transform (denoted by $\mathrm{L}$ ) on both sides of Eq.(4.1), we have

$$
\begin{aligned}
& L\left[\frac{\partial^{2} y}{\partial t^{2}}\right]+L\left[R_{1} y(x, t)+R_{2} y(x, t)\right]+L[N y(x, t)]=L[f(x, t)], \\
& s^{2} L[y(x, t)]-s y(x, 0)-\frac{\partial y}{\partial t}(x, 0)=-L\left[R_{1} y(x, t)+R_{2} y(x, t)\right]+ \\
& -L[N y(x, t)]+L[f(x, t)] .
\end{aligned}
$$

Applying the initial conditions given in Eq.(4.1), we have

$$
\begin{aligned}
& L[y(x, t)]=\frac{\alpha(x)}{s}+\frac{\beta(x)}{s^{2}}-\frac{1}{s^{2}}\left(L\left[R_{1} y(x, t)+R_{2} y(x, t)\right]+L[N y(x, t)]\right)+ \\
& +\frac{1}{s^{2}}(L[f(x, t)]) .
\end{aligned}
$$

Operating the inverse Laplace transform on both sides of Eq.(4.3) gives

$$
y(x, t)=F(x, t)-L^{-1}\left[\frac{1}{s^{2}}\left(L\left[R_{1} y(x, t)+R_{2} y(x, t)\right]+L[N y(x, t)]\right)\right]
$$

where $F(x, t)$ represents the term arising from the source term and the prescribed initial conditions. Now we apply the homotopy perturbation method

$$
y(x, t)=\sum_{n=0}^{\infty} P^{n} y_{n}(x, t),
$$

and the nonlinear term can be decomposed as

$$
N y(x, t)=\sum_{n=0}^{\infty} P^{n} H_{n}(y)
$$


and

$$
H_{n}(y)=\frac{1}{n !} \frac{\partial^{n}}{\partial p^{n}} N\left(\sum_{k=0}^{n} P^{k} y_{k}\right)_{p=0},
$$

coupling the Laplace transform and the homotopy perturbation method gives

$$
\begin{aligned}
& \sum_{n=0}^{\infty} P^{n} y_{n}(x, t)=F(x, t)+ \\
& -p\left(L^{-1}\left[\frac{1}{s^{2}} L\left[\left(R_{1}+R_{2}\right) \sum_{n=0}^{\infty} P^{n} y_{n}(x, t)+\sum_{n=0}^{\infty} P^{n} H_{n}(y)\right]\right]\right)
\end{aligned}
$$

so comparing the coefficients of like powers of $p$, we have the following approximations

$$
\begin{aligned}
& p^{\circ}: y_{\circ}(x, t)=F(x, t), \\
& p^{1}: y_{1}(x, t)=-L^{-1}\left[\frac{1}{s^{2}} L\left[\left(R_{1}+R_{2}\right) y_{\circ}(x, t)+H_{\circ}(y)\right]\right], \\
& p^{2}: y_{2}(x, t)=-L^{-1}\left[\frac{1}{s^{2}} L\left[\left(R_{1}+R_{2}\right) y_{1}(x, t)+H_{1}(y)\right]\right], \\
& p^{3}: y_{3}(x, t)=-L^{-1}\left[\frac{1}{s^{2}} L\left[\left(R_{1}+R_{2}\right) y_{2}(x, t)+H_{2}(y)\right]\right] .
\end{aligned}
$$

Hence, the general solution takes the form

$$
y(x, t)=y_{0}(x, t)+y_{1}(x, t)+y_{2}(x, t)+y_{3}(x, t)+\ldots
$$

\section{The plane Couette flow solution} results

Applying the method to Eqs (4.2)-(4.4) subject to conditions (4.5)-(4.7), we obtain the following

$$
\begin{aligned}
& u_{0}(y, t)=1+\cos (\omega y) \\
& u_{1}(y, t)=-\omega^{2} \cos (\omega y)\left[\delta_{*}+6 \beta \omega^{2}(\sin (\omega y))^{2}\right] t-M[1+\cos (\omega y)] t \\
& u_{2}(y, t)=\frac{\delta_{*}^{2} \omega^{4} \cos (\omega y) t^{2}}{2}+30 \delta_{*} \beta \omega^{6} \cos (\omega y)(\sin (\omega y))^{2} t^{2}+ \\
& -\frac{12 \delta_{*} \beta \omega^{6}(\cos (\omega y))^{3} t^{2}}{2}+\delta_{*} M \omega^{2} \cos (\omega y) t^{2}+\delta_{*} \alpha \omega^{4} \cos (\omega y) t+ \\
& +42 \alpha \beta \omega^{6} \cos (\omega y)(\sin (\omega y))^{2} t-12 \alpha \beta \omega^{6}(\cos (\omega y))^{3} t+\alpha \beta \omega^{2} \cos (\omega y) t+ \\
& -108 \beta^{2} \omega^{8} \cos (\omega y)(\sin (\omega y))^{2} t^{2}+162 \beta^{2} \omega^{8} \cos (\omega y)(\sin (\omega y))^{4} t^{2}+ \\
& +12 \beta M \omega^{4} \cos (\omega y)(\sin (\omega y))^{2} t^{2}+\frac{M^{2}}{2}[1+\cos (\omega y)] t^{2}
\end{aligned}
$$


The solution for the velocity profile of the plane Couette flow is given by $u(y, t)=u_{\mathrm{o}}+u_{1}+u_{2}+u_{3}+\ldots=1+\cos (\omega y)-\omega^{2} \cos (\omega y)\left[\delta_{*}+6 \beta \omega^{2}(\sin (\omega y))^{2}\right] t-M[1+\cos (\omega y)] t+$ $+\frac{\delta_{*}^{2} \omega^{4} \cos (\omega y) t^{2}}{2}+30 \delta_{*} \beta \omega^{6} \cos (\omega y)(\sin (\omega y))^{2} t^{2}-\frac{12 \delta_{*} \beta \omega^{6}(\cos (\omega y))^{3} t^{2}}{2}+\delta_{*} M \omega^{2} \cos (\omega y) t^{2}+$ $+\delta_{*} \alpha \omega^{4} \cos (\omega y) t+42 \alpha \beta \omega^{6} \cos (\omega y)(\sin (\omega y))^{2} t-12 \alpha \beta \omega^{6}(\cos (\omega y))^{3} t+\alpha \beta \omega^{2} \cos (\omega y) t+$ $-108 \beta^{2} \omega^{8} \cos (\omega y)(\sin (\omega y))^{2} t^{2}+162 \beta^{2} \omega^{8} \cos (\omega y)(\sin (\omega y))^{4} t^{2}+12 \beta M \omega^{4} \cos (\omega y)(\sin (\omega y))^{2} t^{2}+$ $+\frac{M^{2}}{2}[1+\cos (\omega y)] t^{2}-\frac{\delta_{*}^{3} \omega^{6} \cos (\omega y) t^{3}}{6}+\frac{156 \delta_{*}^{2} \beta \omega^{8}(\cos (\omega y))^{3} t^{3}}{6}-91 \delta_{*}^{2} \beta \omega^{8} \cos (\omega y)(\sin (\omega y))^{2} t^{3}+$ $-\frac{\delta_{*}^{2} M \omega^{4} \cos (\omega y) t^{3}}{2}-\delta_{*}^{2} \alpha \omega^{6} \cos (\omega y) t^{2}+138 \delta_{*} \alpha \beta \omega^{8}(\cos (\omega y))^{3} t^{2}-438 \delta_{*} \alpha \beta \omega^{8} \cos (\omega y)(\sin (\omega y))^{2} t^{2}+$ $-2 \delta_{*} \alpha M \omega^{4} \cos (\omega y) t^{2}-\frac{432 \delta_{*} \beta^{2} \omega^{10}(\cos (\omega y))^{5} t^{3}}{6}+1872 \delta_{*} \beta^{2} \omega^{10}(\cos (\omega y))^{3}(\sin (\omega y))^{2} t^{3}+$

$-1746 \delta_{*} \beta^{2} \omega^{10} \cos (\omega y)(\sin (\omega y))^{4} t^{3}+10 \delta_{*} \beta M \omega^{6}(\cos (\omega y))^{3} t^{3}-56 \delta_{*} \beta M \omega^{6} \cos (\omega y)(\sin (\omega y))^{2} t^{3}+$ $-\frac{\delta_{*} M^{2} \omega^{2} \cos (\omega y) t^{3}}{2}-\delta_{*} \alpha^{2} \omega^{6} \cos (\omega y) t+120 \alpha^{2} \beta \omega^{8}(\cos (\omega y))^{3} t-366 \alpha^{2} \beta \omega^{8} \cos (\omega y)(\sin (\omega y))^{2} t+$ $-\alpha^{2} M \omega^{4} \cos (\omega y) t-\frac{432 \alpha \beta^{2} \omega^{10}(\cos (\omega y))^{5} t^{2}}{2}+4860 \alpha \beta^{2} \omega^{10}(\cos (\omega y))^{3}(\sin (\omega y))^{2} t^{2}+$ $-4104 \alpha \beta^{2} \omega^{10} \cos (\omega y)(\sin (\omega y))^{4} t^{2}+30 \alpha \beta M \omega^{6}(\cos (\omega y))^{3} t^{2}-114 \alpha \beta M \omega^{6} \cos (\omega y)(\sin (\omega y))^{2} t^{2}+$ $-\alpha M^{2} \omega^{2} \cos (\omega y) t^{2}+$

$-\frac{12960 \beta^{3} \omega^{12}(\cos (\omega y))^{5}(\sin (\omega y))^{2} t^{3}}{6}+\frac{84240 \beta^{3} \omega^{12}(\cos (\omega y))^{3}(\sin (\omega y))^{4} t^{3}}{6}-\frac{43416 \beta^{3} \omega^{12} \cos (\omega y)(\sin (\omega y))^{6} t^{3}}{6}+$ $+324 \beta^{2} M \omega^{8}(\cos (\omega y))^{3}(\sin (\omega y))^{2} t^{3}-486 \beta^{2} M \omega^{8} \cos (\omega y)(\sin (\omega y))^{4} t^{3}-13 \beta M^{2} \omega^{4} \cos (\omega y)(\sin (\omega y))^{2} t^{3}+$ $-\frac{M^{2}}{6}[1+\cos (\omega y)] t^{3}+\ldots$

For the temperature profile of the plane Couette flow we have

$$
\begin{aligned}
& \theta_{0}(y, t)=1+\cos (\omega y)+\delta_{*} \mathrm{Ec} \omega^{2}(\sin (\omega y))^{2} t+\beta \mathrm{Ec} \omega^{4}(\sin (\omega y))^{4} t, \\
& \theta_{1}(y, t)=-\frac{\omega^{2}\left[\delta_{*}+R\right] \cos (\omega y) t}{\operatorname{Pr}}-\frac{2 \delta_{*} \mathrm{Ec} \omega^{4}\left[\delta_{*}+R\right](\sin (\omega y))^{2} t^{2}}{2 \operatorname{Pr}}+ \\
& +\frac{2 \delta_{*} \mathrm{Ec} \omega^{4}\left[\delta_{*}+R\right](\cos (\omega y))^{2} t^{2}}{2 \operatorname{Pr}}-\frac{4 \beta E c \omega^{6}\left[\delta_{*}+R\right](\sin (\omega y))^{4} t^{2}}{2 \operatorname{Pr}}+ \\
& +\frac{12 \beta \mathrm{Ec} \omega^{6}\left[\delta_{*}+R\right](\cos (\omega y))^{2}(\sin (\omega y))^{2} t^{2}}{2 \operatorname{Pr}},
\end{aligned}
$$




$$
\begin{aligned}
& \theta_{2}(y, t)=\frac{\omega^{4}\left[\delta_{*}+R\right]^{2} \cos (\omega y) t^{2}}{2 \operatorname{Pr}}-\frac{8 \delta_{*} \mathrm{Ec} \omega^{6}\left[\delta_{*}+R\right]^{2}(\cos (\omega y))^{2} t^{3}}{6 \operatorname{Pr}^{2}}+ \\
& +\frac{8 \delta_{*} \mathrm{Ec} \omega^{6}\left[\delta_{*}+R\right]^{2}(\sin (\omega y))^{2} t^{3}}{6 \operatorname{Pr}^{2}}+\frac{24 \beta \mathrm{Ec} \omega^{8}\left[\delta_{*}+R\right]^{2}(\cos (\omega y))^{4} t^{3}}{6 \operatorname{Pr}^{2}}+ \\
& -\frac{192 \beta \operatorname{Ec} \omega^{8}\left[\delta_{*}+R\right]^{2}(\cos (\omega y))^{2}(\sin (\omega y))^{2} t^{3}}{6 \operatorname{Pr}^{2}}+ \\
& \frac{40 \beta E c \omega^{8}\left[\delta_{*}+R\right]^{2}(\sin (\omega y))^{4} t^{2}}{6 \operatorname{Pr}^{2}} .
\end{aligned}
$$

The solution for the temperature profile of the plane Couette flow is given by

$$
\begin{aligned}
& \theta(y, t)=\theta_{0}+\theta_{1}+\theta_{2}+\theta_{3}+\cdots, \\
& \theta(y, t)=1+\cos (\omega y)+\delta * \operatorname{Ec} \omega^{2}(\sin (\omega y))^{2} t+\beta E \mathrm{Ec} \omega^{4}(\sin (\omega y))^{4} t+ \\
& -\frac{\omega^{2}\left[\delta_{*}+R\right] \cos (\omega y) t}{\operatorname{Pr}}-\frac{2 \delta_{*} \operatorname{Ec} \omega^{4}\left[\delta_{*}+R\right](\sin (\omega y))^{2} t^{2}}{2 \operatorname{Pr}}+ \\
& +\frac{2 \delta_{*} \mathrm{Ec} \omega^{4}\left[\delta_{*}+R\right](\cos (\omega y))^{2} t^{2}}{2 \operatorname{Pr}}-\frac{4 \beta \mathrm{Ec} \omega^{6}\left[\delta_{*}+R\right](\sin (\omega y))^{4} t^{2}}{2 \operatorname{Pr}}+ \\
& +\frac{12 \beta \mathrm{Ec} \omega^{6}\left[\delta_{*}+R\right](\cos (\omega y))^{2}(\sin (\omega y))^{2} t^{2}}{2 \operatorname{Pr}}+ \\
& \frac{\omega^{4}\left[\delta_{*}+R\right]^{2} \cos (\omega y) t^{2}}{2 \operatorname{Pr}}-\frac{8 \delta_{*} E \mathrm{Ec} \omega^{6}\left[\delta_{*}+R\right]^{2}(\cos (\omega y))^{2} t^{3}}{6 \operatorname{Pr}^{2}}+ \\
& +\frac{8 \delta_{*} \mathrm{Ec} \omega^{6}\left[\delta_{*}+R\right]^{2}(\sin (\omega y))^{2} t^{3}}{6 \operatorname{Pr}^{2}}+\frac{24 \beta \mathrm{Ec} \omega^{8}\left[\delta_{*}+R\right]^{2}(\cos (\omega y))^{4} t^{3}}{6 \operatorname{Pr}^{2}}+ \\
& -\frac{192 \beta \mathrm{Ec} \omega^{8}\left[\delta_{*}+R\right]^{2}(\cos (\omega y))^{2}(\sin (\omega y))^{2} t^{3}}{6 \operatorname{Pr}^{2}}+ \\
& \frac{40 \beta \mathrm{Ec} \omega^{8}\left[\delta_{*}+R\right]^{2}(\sin (\omega y))^{4} t^{2}}{6 \operatorname{Pr}^{2}}-\frac{\omega^{6}\left[\delta_{*}+R\right]^{3} \cos (\omega y) t^{3}}{6 \operatorname{Pr}^{3}}+ \\
& +\frac{16 \delta_{*} \mathrm{Ec} \omega^{8}\left[\delta_{*}+R\right]^{3}(\cos (\omega y))^{2} t^{4}}{24 \mathrm{Pr}^{3}}-\frac{16 \delta_{*} \mathrm{Ec} \omega^{8}\left[\delta_{*}+R\right]^{3}(\sin (\omega y))^{2} t^{4}}{24 \operatorname{Pr}^{3}}+ \\
& -\frac{480 \beta \mathrm{Ec} \omega^{10}\left[\delta_{*}+R\right]^{3}(\cos (\omega y))^{4} t^{4}}{24 \operatorname{Pr}^{3}}+ \\
& \frac{3072 \beta \mathrm{Ec} \omega^{10}\left[\delta_{*}+R\right]^{3}(\cos (\omega y))^{2}(\sin (\omega y))^{2} t^{4}}{24 \mathrm{Pr}^{3}}-\frac{544 \beta \mathrm{Ec} \omega^{10}\left[\delta_{*}+R\right]^{3}(\sin (\omega y))^{4} t^{4}}{24 \mathrm{Pr}^{3}}
\end{aligned}
$$

For the concentration profile of the plane Couette flow we have

$$
C_{0}(y, t)=1+\cos (\omega y)-\frac{K r_{*} N c t}{\mathrm{Sc}},
$$




$$
\begin{aligned}
& C_{1}(y, t)=-\frac{\delta_{*} \omega^{2} \cos (\omega y) t}{\mathrm{Sc}}-\frac{K r_{*} \cos (\omega y) t}{\mathrm{Sc}}-\frac{K r_{*} t}{\mathrm{Sc}}+\frac{K r_{*}^{2} N c t^{2}}{2 \mathrm{Sc}^{2}}, \\
& C_{2}(y, t)=\frac{\delta_{*}^{2} \omega^{4} \cos (\omega y) t^{2}}{2 \mathrm{Sc}^{2}}+\frac{\delta_{*} K r_{*} \omega^{2} \cos (\omega y) t^{2}}{2 \mathrm{Sc}^{2}}+ \\
& +\frac{\delta_{*} K r_{*} \omega^{2} \cos (\omega y) t^{2}}{2 \mathrm{Sc}^{2}}+\frac{K r_{*}^{2} t^{2}}{2 \mathrm{Sc}^{2}}+\frac{K r_{*}^{2} \cos (\omega y) t^{2}}{2 \mathrm{Sc}^{2}}-\frac{K r_{*}^{3} N c t^{3}}{6 \mathrm{Sc}^{3}}
\end{aligned}
$$

The solution for the concentration profile of the plane Couette flow is given by

$$
\begin{aligned}
& C(y, t)=C_{0}+C_{1}+C_{2}+C_{3}+C_{4}+\cdots, \\
& C(y, t)=1+\cos (\omega y)-\frac{K r_{*} N c t}{\mathrm{Sc}}-\frac{\delta * \omega^{2} \cos (\omega y) t}{\mathrm{Sc}}-\frac{K r_{*} \cos (\omega y) t}{\mathrm{Sc}}-\frac{K r_{*} t}{\mathrm{Sc}}+\frac{K r_{*}^{2} N c t^{2}}{2 \mathrm{Sc}^{2}}+ \\
& +\frac{\delta_{*}^{2} \omega^{4} \cos (\omega y) t^{2}}{2 \mathrm{Sc}^{2}}+\frac{\delta * K r_{*} \omega^{2} \cos (\omega y) t^{2}}{\mathrm{Sc}^{2}}+\frac{K r_{*}^{2} t^{2}}{2 \mathrm{Sc}^{2}}+\frac{K r_{*}^{2} \cos (\omega y) t^{2}}{2 \mathrm{Sc}^{2}}-\frac{K r_{*}^{3} N c t^{3}}{6 \mathrm{Sc}^{3}}+ \\
& -\frac{\delta_{*}^{3} \omega^{6} \cos (\omega y) t^{3}}{6 \mathrm{Sc}^{3}}-\frac{\delta_{*}^{2} K r_{*} \omega^{4} \cos (\omega y) t^{3}}{3 \mathrm{Sc}^{3}}-\frac{\delta * K r_{*}^{2} \omega^{2} \cos (\omega y) t^{3}}{2 \mathrm{Sc}^{3}}+ \\
& -\frac{K r_{*}^{3} t^{3}}{6 \mathrm{Sc}^{3}}-\frac{K r_{*}^{3} \cos (\omega y) t^{3}}{6 \mathrm{Sc}^{3}}+\frac{K r_{*}^{4} N c t^{4}}{24 \mathrm{Sc}^{4}} .
\end{aligned}
$$

\section{Formulation of the plane Poiseuille flow problem}

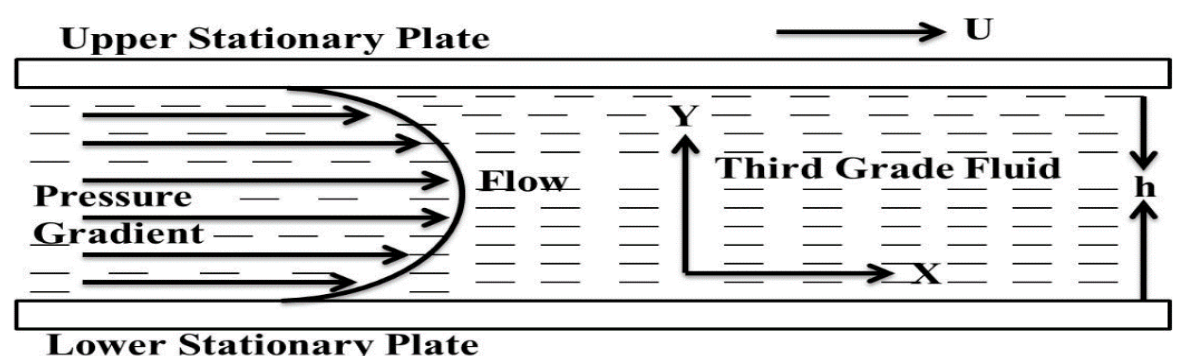

Fig.2. Model of the plane Poiseuille flow.

In the plane Poiseuille flow both plates are stationary whereby the flow between the plates is maintained due to the pressure gradient. For the plane Poiseuille flow with initial and boundary conditions from Eqs (2.2), (3.6) and (3.8) with Eqs (3.12), (3.13) and (3.14) we have

$$
\begin{aligned}
& \rho \frac{\partial u}{\partial t}=-\frac{\partial P}{\partial y}+\mu\left(\frac{\partial^{2} u}{\partial y^{2}}\right)+\alpha_{1}\left(\frac{\partial^{3} u}{\partial y^{2} \partial t}\right)+6 \beta_{3}\left(\frac{\partial u}{\partial y}\right)^{2} \frac{\partial^{2} u}{\partial y^{2}}-\sigma B_{0}^{2} u, \\
& \rho C_{p} \frac{\partial \theta}{\partial t}=k\left(\frac{\partial^{2} \theta}{\partial y^{2}}\right)+\mu\left(\frac{\partial u}{\partial y}\right)^{2}+\alpha_{1} \frac{\partial^{3} u}{\partial y^{2} \partial t}+2 \beta_{3}\left(\frac{\partial u}{\partial y}\right)^{4}+\frac{16 \sigma^{*} \theta_{0}^{3}}{3 k^{*}} \frac{\partial^{2} \theta}{\partial y^{2}}
\end{aligned}
$$




$$
\begin{aligned}
& \frac{\partial C}{\partial t}=D\left(\frac{\partial^{2} C}{\partial y^{2}}\right)-K_{r} C, \\
& u(h, t)=U \cos (\omega y),(y>0), \quad u(0, t)=U \cos (\omega t),(t>0), \\
& u(y, t)=0, \quad \text { at } y \rightarrow \infty(t>0), \\
& \theta(h, t)=\left(\theta_{1}-\theta_{0}\right) \cos (\omega y)+\theta_{0},(y>0), \\
& \theta(0, t)=\left(\theta_{1}-\theta_{0}\right) \cos (\omega t)+\theta_{0},(t>0), \\
& C(h, t)=\left(C_{1}-C_{0}\right) \cos (\omega y)+C_{0},(y>0), \\
& C(0, t)=\left(C_{1}-C_{0}\right) \cos (\omega t)+C_{0},(t>0) .
\end{aligned}
$$

Here $\omega$ is the frequency of the oscillating plate. The dimensionless forms of Eqs (6.1), (6.2) and (6.3) with their initial and boundary conditions (6.4), (6.5) and (6.6) using Eq.(3.7) where for simplicity we drop the bars yields

$$
\begin{aligned}
& \frac{\partial u}{\partial t}=\delta_{*}\left(\frac{\partial^{2} u}{\partial y^{2}}\right)+\alpha\left(\frac{\partial^{3} u}{\partial y^{2} \partial t}\right)+6 \beta\left(\frac{\partial u}{\partial y}\right)^{2}\left(\frac{\partial^{2} u}{\partial y^{2}}\right)-M u-\Omega \\
& \frac{\partial \theta}{\partial t}=\frac{\delta_{*}}{\operatorname{Pr}}\left(\frac{\partial^{2} \theta}{\partial y^{2}}\right)+\delta_{*} \operatorname{Ec}\left(\frac{\partial u}{\partial y}\right)^{2}+\alpha \operatorname{Ec} \frac{\partial^{3} u}{\partial y^{2} \partial t}+\beta \operatorname{Ec}\left(\frac{\partial u}{\partial y}\right)^{4}+\frac{R}{\operatorname{Pr}} \frac{\partial^{2} \theta}{\partial y^{2}} \\
& \frac{\partial C}{\partial t}=\frac{\delta_{*}}{\operatorname{Sc}}\left(\frac{\partial^{2} C}{\partial y^{2}}\right)-\frac{K r_{*}}{\operatorname{Sc}}(C+N c) \\
& u(y, 0)=\cos (\omega y), \quad u(0, t)=\cos (\omega t) \\
& \theta(y, 0)=\cos (\omega y), \quad \theta(\infty, t)=\cos (\omega t) \\
& C(y, 0)=\cos (\omega y), \quad C(0, t)=\cos (\omega t)
\end{aligned}
$$

where $\Omega=\frac{\delta^{2}}{\mu U h} \frac{\partial P}{\partial y}$ is the pressure gradient.

For the plane Poiseuille flow solution we have

$$
\begin{aligned}
& u_{0}(y, t)=\cos (\omega y)-\Omega t \\
& u_{1}(y, t)=-\omega^{2} \cos (\omega y)\left[\delta_{*}+6 \beta \omega^{2}(\sin (\omega y))^{2}\right] t-M \cos (\omega y) t+\frac{M \Omega t^{2}}{2},
\end{aligned}
$$


$u_{2}(y, t)=\frac{\delta_{*}^{2} \omega^{4} \cos (\omega y) t^{2}}{2}+30 \delta_{*} \beta \omega^{6} \cos (\omega y)(\sin (\omega y))^{2} t^{2}-\frac{12 \delta_{*} \beta \omega^{6}(\cos (\omega y))^{3} t^{2}}{2}+$

$+\delta_{*} M \omega^{2} \cos (\omega y) t^{2}+\delta_{*} \alpha \omega^{4} \cos (\omega y) t+42 \alpha \beta \omega^{6} \cos (\omega y)(\sin (\omega y))^{2} t+$

$-12 \alpha \beta \omega^{6}(\cos (\omega y))^{3} t+\alpha \beta \omega^{2} \cos (\omega y) t-108 \beta^{2} \omega^{8} \cos (\omega y)(\sin (\omega y))^{2} t^{2}+162 \beta^{2} \omega^{8} \cos (\omega y)(\sin (\omega y))^{4} t^{2}+$

$+12 \beta M \omega^{4} \cos (\omega y)(\sin (\omega y))^{2} t^{2}+\frac{M^{2} \cos (\omega y) t^{2}}{2}-\frac{M^{2} \Omega t^{3}}{6}$.

The solution for the velocity profile of the plane Poiseuille flow is given by $u(y, t)=u_{0}+u_{1}+u_{2}+u_{3}+\ldots=$ $u(y, t)=\cos (\omega y)-\Omega t-\omega^{2} \cos (\omega y)\left[\delta_{*}+6 \beta \omega^{2}(\sin (\omega y))^{2}\right] t-M \cos (\omega y) t+\frac{M \Omega t^{2}}{2} \times$

$\times \frac{\delta_{*}^{2} \omega^{4} \cos (\omega y) t^{2}}{2}+30 \delta_{*} \beta \omega^{6} \cos (\omega y)(\sin (\omega y))^{2} t^{2}-\frac{12 \delta_{*} \beta \omega^{6}(\cos (\omega y))^{3} t^{2}}{2}$

$+\delta_{*} M \omega^{2} \cos (\omega y) t^{2}+\delta_{*} \alpha \omega^{4} \cos (\omega y) t+42 \alpha \beta \omega^{6} \cos (\omega y)(\sin (\omega y))^{2} t+$

$-12 \alpha \beta \omega^{6}(\cos (\omega y))^{3} t+\alpha \beta \omega^{2} \cos (\omega y) t-108 \beta^{2} \omega^{8} \cos (\omega y)(\sin (\omega y))^{2} t^{2}+$

$+162 \beta^{2} \omega^{8} \cos (\omega y)(\sin (\omega y))^{4} t^{2}+12 \beta M \omega^{4} \cos (\omega y)(\sin (\omega y))^{2} t^{2}+\frac{M^{2}}{2}[1+\cos (\omega y)] t^{2}+$

$-\frac{\delta_{*}^{3} \omega^{6} \cos (\omega y) t^{3}}{6}+\frac{156 \delta_{*}^{2} \beta \omega^{8}(\cos (\omega y))^{3} t^{3}}{6}-91 \delta_{*}^{2} \beta \omega^{8} \cos (\omega y)(\sin (\omega y))^{2} t^{3}+$

$-\frac{\delta_{*}^{2} M \omega^{4} \cos (\omega y) t^{3}}{2}-\delta_{*}^{2} \alpha \omega^{6} \cos (\omega y) t^{2}+138 \delta_{*} \alpha \beta \omega^{8}(\cos (\omega y))^{3} t^{2}+$

$-438 \delta_{*} \alpha \beta \omega^{8} \cos (\omega y)(\sin (\omega y))^{2} t^{2}-2 \delta * \alpha M \omega^{4} \cos (\omega y) t^{2}+$

$-\frac{432 \delta_{*} \beta^{2} \omega^{10}(\cos (\omega y))^{5} t^{3}}{6}+1872 \delta_{*} \beta^{2} \omega^{10}(\cos (\omega y))^{3}(\sin (\omega y))^{2} t^{3}+$

$-1746 \delta_{*} \beta^{2} \omega^{10} \cos (\omega y)(\sin (\omega y))^{4} t^{3}+10 \delta_{*} \beta M \omega^{6}(\cos (\omega y))^{3} t^{3}-$

$56 \delta_{*} \beta M \omega^{6} \cos (\omega y)(\sin (\omega y))^{2} t^{3}-\frac{\delta_{*} M^{2} \omega^{2} \cos (\omega y) t^{3}}{2}-\delta_{*} \alpha^{2} \omega^{6} \cos (\omega y) t+$

$+120 \alpha^{2} \beta \omega^{8}(\cos (\omega y))^{3} t-366 \alpha^{2} \beta \omega^{8} \cos (\omega y)(\sin (\omega y))^{2} t-\alpha^{2} M \omega^{4} \cos (\omega y) t+$

$-\frac{432 \alpha \beta^{2} \omega^{10}(\cos (\omega y))^{5} t^{2}}{2}+4860 \alpha \beta^{2} \omega^{10}(\cos (\omega y))^{3}(\sin (\omega y))^{2} t^{2}+$

$-4104 \alpha \beta^{2} \omega^{10} \cos (\omega y)(\sin (\omega y))^{4} t^{2}+30 \alpha \beta M \omega^{6}(\cos (\omega y))^{3} t^{2}-$

$114 \alpha \beta M \omega^{6} \cos (\omega y)(\sin (\omega y))^{2} t^{2}-\alpha M^{2} \omega^{2} \cos (\omega y) t^{2}-$

$\frac{12960 \beta^{3} \omega^{12}(\cos (\omega y))^{5}(\sin (\omega y))^{2} t^{3}}{6}+\frac{84240 \beta^{3} \omega^{12}(\cos (\omega y))^{3}(\sin (\omega y))^{4} t^{3}}{6}+$

$-\frac{43416 \beta^{3} \omega^{12} \cos (\omega y)(\sin (\omega y))^{6} t^{3}}{6}+324 \beta^{2} M \omega^{8}(\cos (\omega y))^{3}(\sin (\omega y))^{2} t^{3}+$

$-486 \beta^{2} M \omega^{8} \cos (\omega y)(\sin (\omega y))^{4} t^{3}-13 \beta M^{2} \omega^{4} \cos (\omega y)(\sin (\omega y))^{2} t^{3}-\frac{M^{2}}{6}[1+\cos (\omega y)] t^{3}+\ldots$ 
For the temperature profile of the plane Poiseuille flow we have

$$
\begin{aligned}
& \theta_{0}(y, t)=\cos (\omega y)+\delta_{*} \operatorname{Ec} \omega^{2}(\sin (\omega y))^{2} t+\beta \operatorname{Ec} \omega^{4}(\sin (\omega y))^{4} t \\
& \theta_{1}(y, t)=-\frac{\omega^{2}\left[\delta_{*}+R\right] \cos (\omega y) t}{\operatorname{Pr}}-\frac{2 \delta_{*} \mathrm{Ec} \omega^{4}\left[\delta_{*}+R\right](\sin (\omega y))^{2} t^{2}}{2 \operatorname{Pr}}+ \\
& +\frac{2 \delta_{*} \operatorname{Ec} \omega^{4}\left[\delta_{*}+R\right](\cos (\omega y))^{2} t^{2}}{2 \operatorname{Pr}}-\frac{4 \beta E c \omega^{6}\left[\delta_{*}+R\right](\sin (\omega y))^{4} t^{2}}{2 \operatorname{Pr}}+ \\
& +\frac{12 \beta E \operatorname{Ec} \omega^{6}\left[\delta_{*}+R\right](\cos (\omega y))^{2}(\sin (\omega y))^{2} t^{2}}{2 \operatorname{Pr}} .
\end{aligned}
$$

The solution for the temperature profile of the plane Poiseuille flow is given by

$$
\begin{aligned}
& \theta(y, t)=\theta_{0}+\theta_{1}+\theta_{2}+\theta_{3}+\cdots \\
& \theta(y, t)=\cos (\omega y)+\delta_{*} \mathrm{Ec} \omega^{2}(\sin (\omega y))^{2} t+\beta \mathrm{Ec} \omega^{4}(\sin (\omega y))^{4} t+ \\
& -\frac{\omega^{2}\left[\delta_{*}+R\right] \cos (\omega y) t}{\operatorname{Pr}}-\frac{2 \delta_{*} \operatorname{Ec} \omega^{4}\left[\delta_{*}+R\right](\sin (\omega y))^{2} t^{2}}{2 \operatorname{Pr}}+ \\
& +\frac{2 \delta_{*} \mathrm{Ec} \omega^{4}\left[\delta_{*}+R\right](\cos (\omega y))^{2} t^{2}}{2 \operatorname{Pr}}-\frac{4 \beta \mathrm{Ec} \omega^{6}\left[\delta_{*}+R\right](\sin (\omega y))^{4} t^{2}}{2 \operatorname{Pr}} \\
& +\frac{12 \beta \mathrm{Ec} \omega^{6}\left[\delta_{*}+R\right](\cos (\omega y))^{2}(\sin (\omega y))^{2} t^{2}}{2 \operatorname{Pr}}+\frac{\omega^{4}\left[\delta_{*}+R\right]^{2} \cos (\omega y) t^{2}}{2 \operatorname{Pr}}+ \\
& -\frac{8 \delta_{*} \mathrm{Ec} \omega^{6}\left[\delta_{*}+R\right]^{2}(\cos (\omega y))^{2} t^{3}}{6 \operatorname{Pr}^{2}}+\frac{8 \delta_{*} \mathrm{Ec} \omega^{6}\left[\delta_{*}+R\right]^{2}(\sin (\omega y))^{2} t^{3}}{6 \operatorname{Pr}^{2}}+ \\
& +\frac{24 \beta \mathrm{Ec} \omega^{8}\left[\delta_{*}+R\right]^{2}(\cos (\omega y))^{4} t^{3}}{6 \operatorname{Pr}^{2}}-\frac{192 \beta \mathrm{Ec} \omega^{8}\left[\delta_{*}+R\right]^{2}(\cos (\omega y))^{2}(\sin (\omega y))^{2} t^{3}}{6 \operatorname{Pr}^{2}}+ \\
& \frac{40 \beta \mathrm{Ec} \omega^{8}\left[\delta_{*}+R\right]^{2}(\sin (\omega y))^{4} t^{2}}{6 \operatorname{Pr}^{2}}-\frac{\omega^{6}\left[\delta_{*}+R\right]^{3} \cos (\omega y) t^{3}}{6 \operatorname{Pr}^{3}}+\frac{16 \delta_{*} \mathrm{Ec} \omega^{8}\left[\delta_{*}+R\right]^{3}(\cos (\omega y))^{2} t^{4}}{24 \operatorname{Pr}^{3}}+ \\
& -\frac{16 \delta_{*} \mathrm{Ec} \omega^{8}\left[\delta_{*}+R\right]^{3}(\sin (\omega y))^{2} t^{4}}{24 \mathrm{Pr}^{3}}-\frac{480 \beta \mathrm{Ec} \omega^{10}\left[\delta_{*}+R\right]^{3}(\cos (\omega y))^{4} t^{4}}{24 \mathrm{Pr}^{3}}+ \\
& \frac{3072 \beta \mathrm{Ec} \omega^{10}\left[\delta_{*}+R\right]^{3}(\cos (\omega y))^{2}(\sin (\omega y))^{2} t^{4}}{24 \mathrm{Pr}^{3}}-\frac{544 \beta \mathrm{Ec} \omega^{10}\left[\delta_{*}+R\right]^{3}(\sin (\omega y))^{4} t^{4}}{24 \mathrm{Pr}^{3}} .
\end{aligned}
$$

For the concentration profile of the plane Poiseuille flow we have

$$
\begin{aligned}
& C_{0}(y, t)=\cos (\omega y)-\frac{K r_{*} N c t}{\mathrm{Sc}}, \\
& C_{1}(y, t)=-\frac{\delta_{*} \omega^{2} \cos (\omega y) t}{\mathrm{Sc}}-\frac{K r_{*} \cos (\omega y) t}{\mathrm{Sc}}+\frac{K r_{*}^{2} N c t^{2}}{2 \mathrm{Sc}^{2}},
\end{aligned}
$$




$$
\begin{aligned}
& C_{2}(y, t)=\frac{\delta_{*}^{2} \omega^{4} \cos (\omega y) t^{2}}{2 \mathrm{Sc}^{2}}+\frac{\delta_{*} K r_{*} \omega^{2} \cos (\omega y) t^{2}}{2 \mathrm{Sc}^{2}}+ \\
& +\frac{\delta_{*} K r_{*} \omega^{2} \cos (\omega y) t^{2}}{2 \mathrm{Sc}^{2}}+\frac{K r_{*}^{2} \cos (\omega y) t^{2}}{2 \mathrm{Sc}^{2}}-\frac{K r_{*}^{3} N c t^{3}}{6 \mathrm{Sc}^{3}}
\end{aligned}
$$

The solution for the concentration profile of the plane Poiseuille flow is given by

$$
\begin{aligned}
& C(y, t)=C_{0}+C_{1}+C_{2}+C_{3}+C_{4}+\cdots \\
& C(y, t)=\cos (\omega y)-\frac{K r_{*} N c t}{\mathrm{Sc}}-\frac{\delta_{*} \omega^{2} \cos (\omega y) t}{\mathrm{Sc}}-\frac{K r_{*} \cos (\omega y) t}{\mathrm{Sc}}+\frac{K r_{*}^{2} N c t^{2}}{2 \mathrm{Sc}^{2}}+ \\
& +\frac{\delta_{*}^{2} \omega^{4} \cos (\omega y) t^{2}}{2 \mathrm{Sc}^{2}}+\frac{\delta_{*} K r_{*} \omega^{2} \cos (\omega y) t^{2}}{\mathrm{Sc}^{2}}+\frac{K r_{*}^{2} \cos (\omega y) t^{2}}{2 \mathrm{Sc}^{2}}-\frac{K r_{*}^{3} N c t^{3}}{6 \mathrm{Sc}^{3}}+ \\
& -\frac{\delta_{*}^{3} \omega^{6} \cos (\omega y) t^{3}}{6 \mathrm{Sc}^{3}}-\frac{\delta_{*}^{2} K r_{*} \omega^{4} \cos (\omega y) t^{3}}{3 \mathrm{Sc}^{3}}-\frac{\delta * K r_{*}^{2} \omega^{2} \cos (\omega y) t^{3}}{2 \mathrm{Sc}^{3}}-\frac{K r_{*}^{3} \cos (\omega y) t^{3}}{6 \mathrm{Sc}^{3}}+ \\
& +\frac{K r_{*}^{4} N c t^{4}}{24 \mathrm{Sc}^{4}}
\end{aligned}
$$

\section{Formulation of the plane Couette-Poiseuille flow problem}

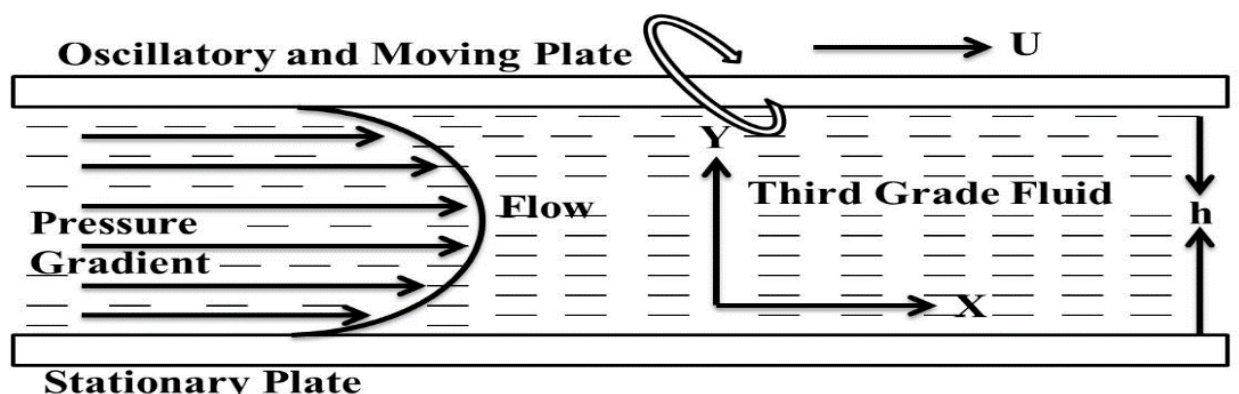

Fig.3. Model of the plane Couette-Poiseuille flow.

Suppose that the fluid is restricted between two horizontal parallel plates. The motion of the fluid depends on the motion of the upper plate and pressure gradient. For the plane Couette-Poiseuille flow with initial and boundary conditions from Eqs (2.2), (3.6) and (3.8) with Eqs (3.12), (3.13) and (3.14) yield we have

$$
\begin{aligned}
& \rho \frac{\partial u}{\partial t}=-\frac{\partial P}{\partial y}+\mu\left(\frac{\partial^{2} u}{\partial y^{2}}\right)+\alpha_{1}\left(\frac{\partial^{3} u}{\partial y^{2} \partial t}\right)+6 \beta_{3}\left(\frac{\partial u}{\partial y}\right)^{2} \frac{\partial^{2} u}{\partial y^{2}}-\sigma B_{0}^{2} u, \\
& \rho C_{p} \frac{\partial \theta}{\partial t}=k\left(\frac{\partial^{2} \theta}{\partial y^{2}}\right)+\mu\left(\frac{\partial u}{\partial y}\right)^{2}+\alpha_{1} \frac{\partial^{3} u}{\partial y^{2} \partial t}+2 \beta_{3}\left(\frac{\partial u}{\partial y}\right)^{4}+\frac{16 \sigma^{*} \theta_{0}^{3}}{3 k^{*}} \frac{\partial^{2} \theta}{\partial y^{2}},
\end{aligned}
$$




$$
\begin{aligned}
& \frac{\partial C}{\partial t}=D\left(\frac{\partial^{2} C}{\partial y^{2}}\right)-K_{r} C, \\
& u(h, t)=U+U \cos (\omega y),(y>0), \quad u(0, t)=U+U \cos (\omega t),(t>0), \\
& u(y, t)=0, \quad \text { at } y \rightarrow \infty(t>0), \\
& \theta(y, t)=\left(\theta_{1}-\theta_{0}\right)+\left(\theta_{1}-\theta_{0}\right) \cos (\omega t)+\theta_{0}, \quad \text { at } \quad y=h(y>0), \\
& \theta(y, t)=\left(\theta_{1}-\theta_{0}\right)+\left(\theta_{1}-\theta_{0}\right) \cos (\omega t)+\theta_{0}, \quad \text { at } y=0(t>0), \\
& C(h, t)=\left(C_{1}-C_{0}\right)+\left(C_{1}-C_{0}\right) \cos (\omega y)+C_{0}, \quad \text { at } y=h(y>0), \\
& C(y, t)=\left(C_{1}-C_{0}\right)+\left(C_{1}-C_{0}\right) \cos (\omega y)+C_{0}, \quad \text { at } y=0(t>0) .
\end{aligned}
$$

The dimensionless forms of Eqs (7.1), (7.2) and (7.3) with their initial and boundary conditions (7.4), (7.5) and (7.6) using Eq.(3.7) where for simplicity we drop the bars yield

$$
\begin{aligned}
& \frac{\partial u}{\partial t}=\delta_{*}\left(\frac{\partial^{2} u}{\partial y^{2}}\right)+\alpha\left(\frac{\partial^{3} u}{\partial y^{2} \partial t}\right)+6 \beta\left(\frac{\partial u}{\partial y}\right)^{2}\left(\frac{\partial^{2} u}{\partial y^{2}}\right)-M u-\Omega \\
& \frac{\partial \theta}{\partial t}=\frac{\delta_{*}}{\operatorname{Pr}}\left(\frac{\partial^{2} \theta}{\partial y^{2}}\right)+\delta_{*} \operatorname{Ec}\left(\frac{\partial u}{\partial y}\right)^{2}+\alpha \operatorname{Ec} \frac{\partial^{3} u}{\partial y^{2} \partial t}+\beta \operatorname{Ec}\left(\frac{\partial u}{\partial y}\right)^{4}+\frac{R}{\operatorname{Pr}} \frac{\partial^{2} \theta}{\partial y^{2}} \\
& \frac{\partial C}{\partial t}=\frac{\delta_{*}}{\operatorname{Sc}}\left(\frac{\partial^{2} C}{\partial y^{2}}\right)-\frac{K r_{*}}{\operatorname{Sc}}(C+N c) \\
& u(y, 0)=1+\cos (\omega y), \quad u(0, t)=1+\cos (\omega t), \quad u(\infty, t)=0 \\
& \theta(y, 0)=1+\cos (\omega y), \quad \theta(0, t)=1+\cos (\omega t) \\
& C(y, 0)=1+\cos (\omega y), \quad C(0, t)=1+\cos (\omega t)
\end{aligned}
$$

For the plane Couette-Poiseuille flow solution we have

$$
\begin{aligned}
& u_{0}(y, t)=1+\cos (\omega y)-\Omega t \\
& u_{1}(y, t)=-\omega^{2} \cos (\omega y)\left[\delta_{*}+6 \beta \omega^{2}(\sin (\omega y))^{2}\right] t-M t-M \cos (\omega y) t+\frac{M \Omega t^{2}}{2},
\end{aligned}
$$


$u_{2}(y, t)=\frac{\delta_{*}^{2} \omega^{4} \cos (\omega y) t^{2}}{2}+30 \delta_{*} \beta \omega^{6} \cos (\omega y)(\sin (\omega y))^{2} t^{2}-\frac{12 \delta * \beta \omega^{6}(\cos (\omega y))^{3} t^{2}}{2}+$

$+\delta_{*} M \omega^{2} \cos (\omega y) t^{2}+\delta_{*} \alpha \omega^{4} \cos (\omega y) t+42 \alpha \beta \omega^{6} \cos (\omega y)(\sin (\omega y))^{2} t+$

$-12 \alpha \beta \omega^{6}(\cos (\omega y))^{3} t+\alpha \beta \omega^{2} \cos (\omega y) t-108 \beta^{2} \omega^{8} \cos (\omega y)(\sin (\omega y))^{2} t^{2}+$

$+162 \beta^{2} \omega^{8} \cos (\omega y)(\sin (\omega y))^{4} t^{2}+12 \beta M \omega^{4} \cos (\omega y)(\sin (\omega y))^{2} t^{2}+\frac{M^{2} t^{2}}{2}+\frac{M^{2} \cos (\omega y) t^{2}}{2}-\frac{M^{2} \Omega t^{3}}{6}$.

The solution for the velocity profile of the plane Couette-Poiseuille flow is given by $u(y, t)=u_{0}+u_{1}+u_{2}+u_{3}+\ldots=$ $u(y, t)=1+\cos (\omega y)-\Omega t-\omega^{2} \cos (\omega y)\left[\delta_{*}+6 \beta \omega^{2}(\sin (\omega y))^{2}\right] t-M \cos (\omega y) t+\frac{M \Omega t^{2}}{2} \times$

$\times \frac{\delta_{*}^{2} \omega^{4} \cos (\omega y) t^{2}}{2}+30 \delta_{*} \beta \omega^{6} \cos (\omega y)(\sin (\omega y))^{2} t^{2}-\frac{12 \delta * \beta \omega^{6}(\cos (\omega y))^{3} t^{2}}{2}$

$+\delta_{*} M \omega^{2} \cos (\omega y) t^{2}+\delta_{*} \alpha \omega^{4} \cos (\omega y) t+42 \alpha \beta \omega^{6} \cos (\omega y)(\sin (\omega y))^{2} t+$

$-12 \alpha \beta \omega^{6}(\cos (\omega y))^{3} t+\alpha \beta \omega^{2} \cos (\omega y) t-108 \beta^{2} \omega^{8} \cos (\omega y)(\sin (\omega y))^{2} t^{2}$

$+162 \beta^{2} \omega^{8} \cos (\omega y)(\sin (\omega y))^{4} t^{2}+12 \beta M \omega^{4} \cos (\omega y)(\sin (\omega y))^{2} t^{2}+\frac{M^{2} \cos (\omega y) t^{2}}{2}+$

$-\frac{M^{2} \Omega t^{3}}{6}-\frac{\delta_{*}^{3} \omega^{6} \cos (\omega y) t^{3}}{6}+\frac{156 \delta_{*}^{2} \beta \omega^{8}(\cos (\omega y))^{3} t^{3}}{6}-91 \delta_{*}^{2} \beta \omega^{8} \cos (\omega y)(\sin (\omega y))^{2} t^{3}+$

$-\frac{\delta_{*}^{2} M \omega^{4} \cos (\omega y) t^{3}}{2}-\delta_{*}^{2} \alpha \omega^{6} \cos (\omega y) t^{2}+138 \delta_{*} \alpha \beta \omega^{8}(\cos (\omega y))^{3} t^{2}-$

$438 \delta_{*} \alpha \beta \omega^{8} \cos (\omega y)(\sin (\omega y))^{2} t^{2}-2 \delta_{*} \alpha M \omega^{4} \cos (\omega y) t^{2}-$

$\frac{432 \delta_{*} \beta^{2} \omega^{10}(\cos (\omega y))^{5} t^{3}}{6}+1872 \delta_{*} \beta^{2} \omega^{10}(\cos (\omega y))^{3}(\sin (\omega y))^{2} t^{3}-$

$1746 \delta_{*} \beta^{2} \omega^{10} \cos (\omega y)(\sin (\omega y))^{4} t^{3}+10 \delta_{*} \beta M \omega^{6}(\cos (\omega y))^{3} t^{3}-$

$56 \delta_{*} \beta M \omega^{6} \cos (\omega y)(\sin (\omega y))^{2} t^{3}-\frac{\delta_{*} M^{2} \omega^{2} \cos (\omega y) t^{3}}{2}-\delta_{*} \alpha^{2} \omega^{6} \cos (\omega y) t+$

$+120 \alpha^{2} \beta \omega^{8}(\cos (\omega y))^{3} t-366 \alpha^{2} \beta \omega^{8} \cos (\omega y)(\sin (\omega y))^{2} t-\alpha^{2} M \omega^{4} \cos (\omega y) t+$

$-\frac{432 \alpha \beta^{2} \omega^{10}(\cos (\omega y))^{5} t^{2}}{2}+4860 \alpha \beta^{2} \omega^{10}(\cos (\omega y))^{3}(\sin (\omega y))^{2} t^{2}+$

$-4104 \alpha \beta^{2} \omega^{10} \cos (\omega y)(\sin (\omega y))^{4} t^{2}+30 \alpha \beta M \omega^{6}(\cos (\omega y))^{3} t^{2}-$

$114 \alpha \beta M \omega^{6} \cos (\omega y)(\sin (\omega y))^{2} t^{2}-\alpha M^{2} \omega^{2} \cos (\omega y) t^{2}-\frac{12960 \beta^{3} \omega^{12}(\cos (\omega y))^{5}(\sin (\omega y))^{2} t^{3}}{6}+$

$+\frac{84240 \beta^{3} \omega^{12}(\cos (\omega y))^{3}(\sin (\omega y))^{4} t^{3}}{6}-\frac{43416 \beta^{3} \omega^{12} \cos (\omega y)(\sin (\omega y))^{6} t^{3}}{6}+$

$+324 \beta^{2} M \omega^{8}(\cos (\omega y))^{3}(\sin (\omega y))^{2} t^{3}-486 \beta^{2} M \omega^{8} \cos (\omega y)(\sin (\omega y))^{4} t^{3}$

$-13 \beta M^{2} \omega^{4} \cos (\omega y)(\sin (\omega y))^{2} t^{3}-\frac{M^{3} t^{3}}{6}-\frac{M^{3} \cos (\omega y) t^{3}}{6}+\frac{M^{3} \Omega t^{4}}{24}$. 
For the temperature profile of the plane Couette-Poiseuille flow we have

$$
\begin{aligned}
& \theta_{0}(y, t)=1+\cos (\omega y)+\delta_{*} \mathrm{Ec} \omega^{2}(\sin (\omega y))^{2} t+\beta \mathrm{Ec} \omega^{4}(\sin (\omega y))^{4} t \\
& \theta_{I}(y, t)=-\frac{\omega^{2}\left[\delta_{*}+R\right] \cos (\omega y) t}{\operatorname{Pr}}-\frac{2 \delta_{*} \mathrm{Ec} \omega^{4}\left[\delta_{*}+R\right](\sin (\omega y))^{2} t^{2}}{2 \operatorname{Pr}}+ \\
& +\frac{2 \delta_{*} \mathrm{Ec} \omega^{4}\left[\delta_{*}+R\right](\cos (\omega y))^{2} t^{2}}{2 \operatorname{Pr}}-\frac{4 \beta E \mathrm{Ec} \omega^{6}\left[\delta_{*}+R\right](\sin (\omega y))^{4} t^{2}}{2 \operatorname{Pr}}+ \\
& +\frac{12 \beta \mathrm{Ec} \omega^{6}\left[\delta_{*}+R\right](\cos (\omega y))^{2}(\sin (\omega y))^{2} t^{2}}{2 \operatorname{Pr}} .
\end{aligned}
$$

The solution for the temperature profile of the plane Couette-Poiseuille flow is given by

$$
\begin{aligned}
& \theta(y, t)=\theta_{0}+\theta_{1}+\theta_{2}+\theta_{3}+\cdots \\
& \theta(y, t)=1+\cos (\omega y)+\delta_{*} \operatorname{Ec} \omega^{2}(\sin (\omega y))^{2} t+\beta E c \omega^{4}(\sin (\omega y))^{4} t+ \\
& -\frac{\omega^{2}\left[\delta_{*}+R\right] \cos (\omega y) t}{\operatorname{Pr}}-\frac{2 \delta_{*} \operatorname{Ec} \omega^{4}\left[\delta_{*}+R\right](\sin (\omega y))^{2} t^{2}}{2 \operatorname{Pr}}+ \\
& +\frac{2 \delta_{*} \operatorname{Ec} \omega^{4}\left[\delta_{*}+R\right](\cos (\omega y))^{2} t^{2}}{2 \operatorname{Pr}}-\frac{4 \beta E c \omega^{6}\left[\delta_{*}+R\right](\sin (\omega y))^{4} t^{2}}{2 \operatorname{Pr}}+
\end{aligned}
$$$$
+\frac{12 \beta \mathrm{Ec} \omega^{6}\left[\delta_{*}+R\right](\cos (\omega y))^{2}(\sin (\omega y))^{2} t^{2}}{2 \operatorname{Pr}} \frac{\omega^{4}\left[\delta_{*}+R\right]^{2} \cos (\omega y) t^{2}}{2 \operatorname{Pr}}+
$$$$
-\frac{8 \delta_{*} \mathrm{Ec} \omega^{6}\left[\delta_{*}+R\right]^{2}(\cos (\omega y))^{2} t^{3}}{6 \mathrm{Pr}^{2}}+\frac{8 \delta_{*} \mathrm{Ec} \omega^{6}\left[\delta_{*}+R\right]^{2}(\sin (\omega y))^{2} t^{3}}{6 \operatorname{Pr}^{2}}+
$$$$
+\frac{24 \beta \mathrm{Ec} \omega^{8}\left[\delta_{*}+R\right]^{2}(\cos (\omega y))^{4} t^{3}}{6 \operatorname{Pr}^{2}}-\frac{192 \beta \mathrm{Ec} \omega^{8}\left[\delta_{*}+R\right]^{2}(\cos (\omega y))^{2}(\sin (\omega y))^{2} t^{3}}{6 \operatorname{Pr}^{2}}+
$$$$
\frac{40 \beta \mathrm{Ec} \omega^{8}\left[\delta_{*}+R\right]^{2}(\sin (\omega y))^{4} t^{2}}{6 \operatorname{Pr}^{2}}-\frac{\omega^{6}\left[\delta_{*}+R\right]^{3} \cos (\omega y) t^{3}}{6 \operatorname{Pr}^{3}}+\frac{16 \delta_{*} \mathrm{Ec} \omega^{8}\left[\delta_{*}+R\right]^{3}(\cos (\omega y))^{2} t^{4}}{24 \operatorname{Pr}^{3}}+
$$$$
-\frac{16 \delta_{*} \mathrm{Ec} \omega^{8}\left[\delta_{*}+R\right]^{3}(\sin (\omega y))^{2} t^{4}}{24 \mathrm{Pr}^{3}}-\frac{480 \beta \mathrm{Ec} \omega^{10}\left[\delta_{*}+R\right]^{3}(\cos (\omega y))^{4} t^{4}}{24 \mathrm{Pr}^{3}}+
$$$$
\frac{3072 \beta \mathrm{Ec} \omega^{10}\left[\delta_{*}+R\right]^{3}(\cos (\omega y))^{2}(\sin (\omega y))^{2} t^{4}}{24 \mathrm{Pr}^{3}}-\frac{544 \beta \mathrm{Ec} \omega^{10}\left[\delta_{*}+R\right]^{3}(\sin (\omega y))^{4} t^{4}}{24 \mathrm{Pr}^{3}} \text {. }
$$

For the concentration profile of the plane Couette-Poiseuille flow we have

$$
\begin{aligned}
& C_{0}(y, t)=1+\cos (\omega y)-\frac{K r_{*} N c t}{\mathrm{Sc}}, \\
& C_{1}(y, t)=-\frac{\delta_{*} \omega^{2} \cos (\omega y) t}{\mathrm{Sc}}-\frac{K r_{*} \cos (\omega y) t}{\mathrm{Sc}}-\frac{K r_{*} t}{\mathrm{Sc}}+\frac{K r_{*}^{2} N c t^{2}}{2 \mathrm{Sc}^{2}},
\end{aligned}
$$




$$
\begin{aligned}
& C_{2}(y, t)=\frac{\delta_{*}^{2} \omega^{4} \cos (\omega y) t^{2}}{2 \mathrm{Sc}^{2}}+\frac{\delta_{*} K r_{*} \omega^{2} \cos (\omega y) t^{2}}{2 \mathrm{Sc}^{2}}+\frac{\delta_{*} K r_{*} \omega^{2} \cos (\omega y) t^{2}}{2 \mathrm{Sc}^{2}}+ \\
& +\frac{K r_{*}^{2} t^{2}}{2 \mathrm{Sc}^{2}}+\frac{K r_{*}^{2} \cos (\omega y) t^{2}}{2 \mathrm{Sc}^{2}}-\frac{K r_{*}^{3} N c t^{3}}{6 \mathrm{Sc}^{3}}
\end{aligned}
$$

The solution for the concentration profile of the plane Couette-Poiseuille flow is given by

$$
\begin{aligned}
& C(y, t)=C_{0}+C_{1}+C_{2}+C_{3}+C_{4}+\cdots, \\
& C(y, t)=1+\cos (\omega y)-\frac{K r_{*} N c t}{\mathrm{Sc}}-\frac{\delta_{*} \omega^{2} \cos (\omega y) t}{\mathrm{Sc}}-\frac{K r_{*} \cos (\omega y) t}{\mathrm{Sc}}-\frac{K r_{*} t}{\mathrm{Sc}}+ \\
& +\frac{K r_{*}^{2} N c t^{2}}{2 \mathrm{Sc}^{2}}+\frac{\delta_{*}^{2} \omega^{4} \cos (\omega y) t^{2}}{2 \mathrm{Sc}^{2}}+\frac{\delta * K r_{*} \omega^{2} \cos (\omega y) t^{2}}{\mathrm{Sc}^{2}}+\frac{K r_{*}^{2} t^{2}}{2 \mathrm{Sc}^{2}}+ \\
& +\frac{K r_{*}^{2} \cos (\omega y) t^{2}}{2 \mathrm{Sc}^{2}}-\frac{K r_{*}^{3} N c t^{3}}{6 \mathrm{Sc}^{3}}-\frac{\delta_{*}^{3} \omega^{6} \cos (\omega y) t^{3}}{6 \mathrm{Sc}^{3}}-\frac{\delta_{*}^{2} K r_{*} \omega^{4} \cos (\omega y) t^{3}}{3 \mathrm{Sc}^{3}}+ \\
& -\frac{\delta_{*} K r_{*}^{2} \omega^{2} \cos (\omega y) t^{3}}{2 \mathrm{Sc}^{3}}-\frac{K r_{*}^{3} t^{3}}{6 \mathrm{Sc}^{3}}-\frac{K r_{*}^{3} \cos (\omega y) t^{3}}{6 \mathrm{Sc}^{3}}+\frac{K r_{*}^{4} N c t^{4}}{24 \mathrm{Sc}^{4}}
\end{aligned}
$$

\section{Discussion of results}

Systems of non-linear differential Eqs (4.2)-(4.4), (6.15)-(6.17) and (7.15)-(7.17) subject to the conditions (4.5)-(4.7), (6.18)-(6.20) and (7.18)-(7.21) were solved numerically using the He-Laplace method where the effects of various important physical parameters such as the second grade parameter $\alpha$, third grade parameter $\beta$, magnetic field parameter $M$, Prandtl number Pr, Eckert number Ec, thermal radiation parameter $R$, Schmidt number Sc, chemical reaction parameter $K r *$ and pressure gradient $\Omega$ on non-dimensional velocity components, temperature and concentration components for the three unsteady flow problems, namely: the Couette flow, Poiseuille flow and Couette-Poiseuille flow, were analyzed and discussed in detail.

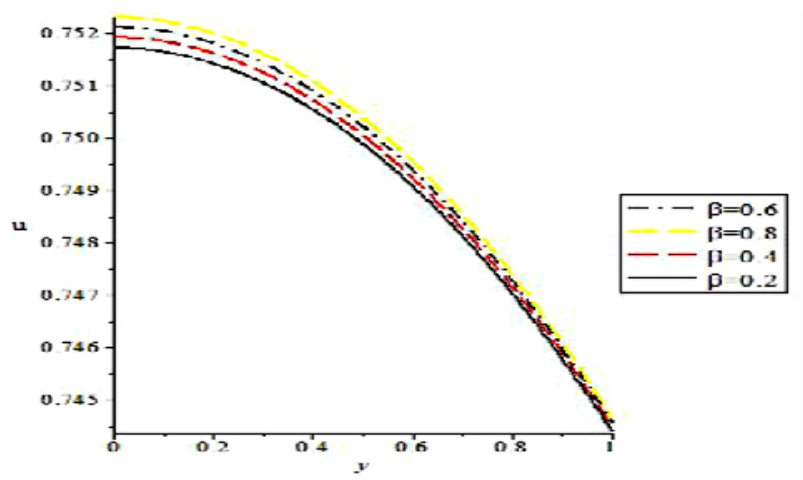

Fig.4. Effect of $\beta$ on the Couette flow velocity profile $M=0.5, t=1.5, \alpha=0.3, \delta_{*}=1, \omega=0.2$.

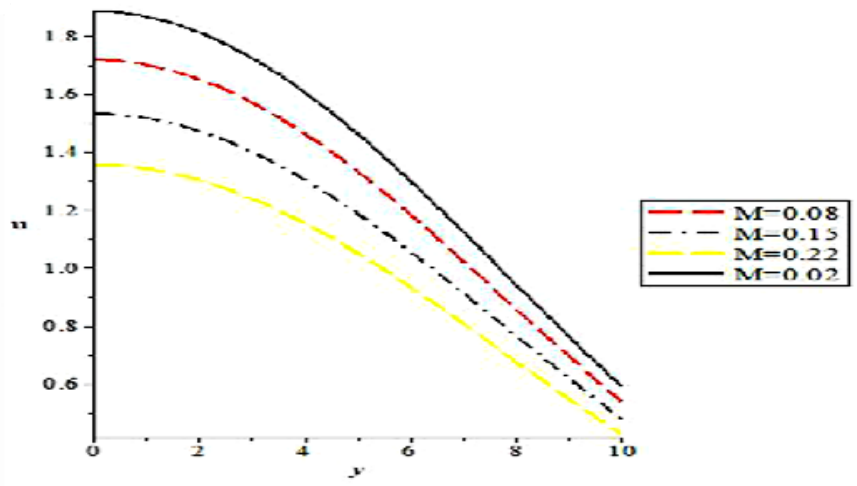

Fig.5. Effect of $M$ on Couette flow velocity profile when $\beta=0.5, t=1.5, \alpha=0.3, \delta_{*}=1, \omega=0.2$. 

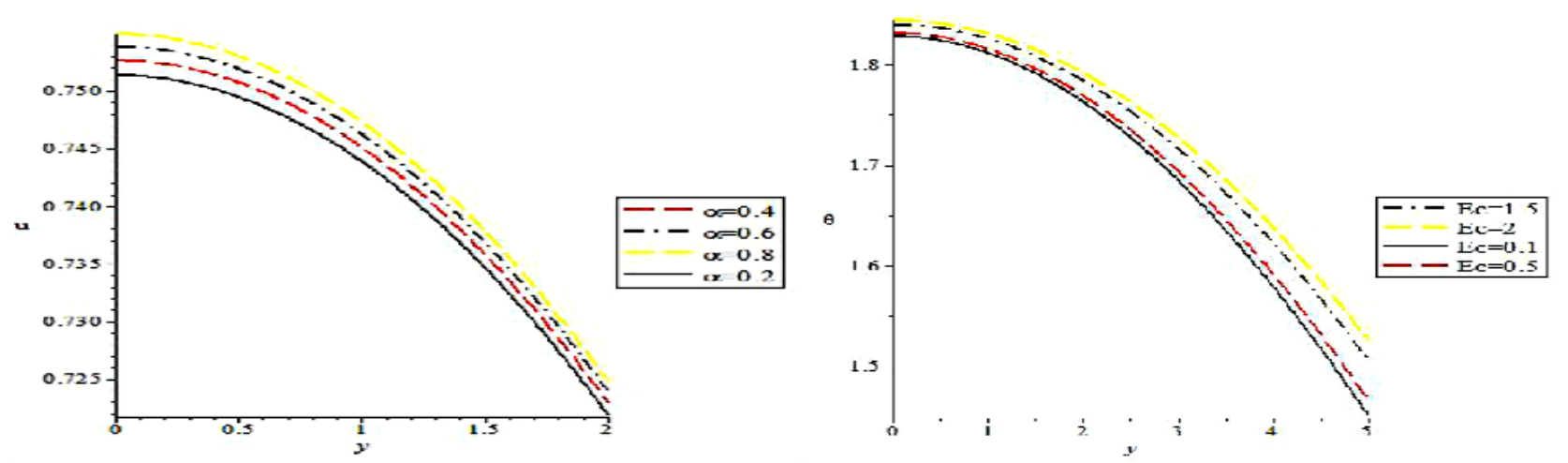

Fig.6. Effect of $\alpha$ on the Couette flow velocity Fig.7. Effect of Ec on the Couette flow temperature profile when $\beta=0.5, M=0.5, t=1.5, \delta_{*}=1, \omega=0.2$. profile when $\beta=0.3, \quad \operatorname{Pr}=0.5, \quad t=1.5, \quad R=0.5$, $\delta_{*}=1, \omega=0.2$.
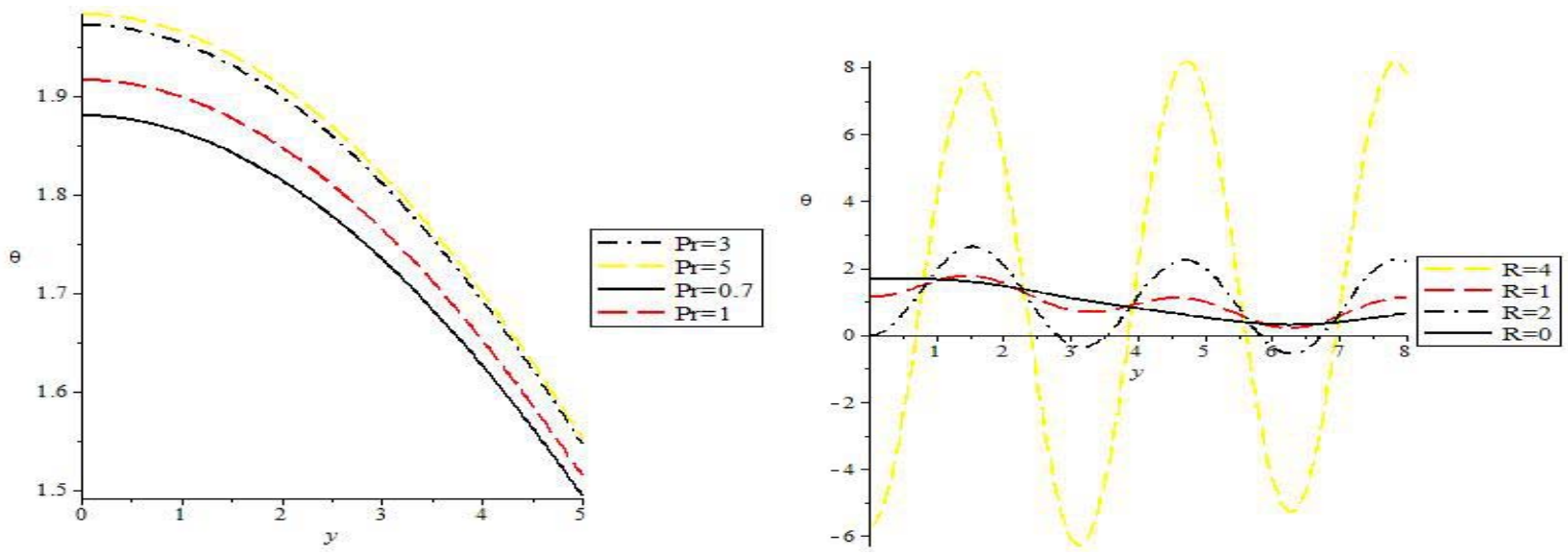

Fig.8. Effect of Pr on the Couette flow temperature Fig.9. Effect of $R$ on the Couette flow temperature profile when $\beta=0.3, \mathrm{Ec}=0.5, t=1.5, R=0.5$, $\delta_{*}=1, \omega=0.2$. profile when $\beta=0.3, \mathrm{Ec}=0.2, t=1.5, \operatorname{Pr}=1$,

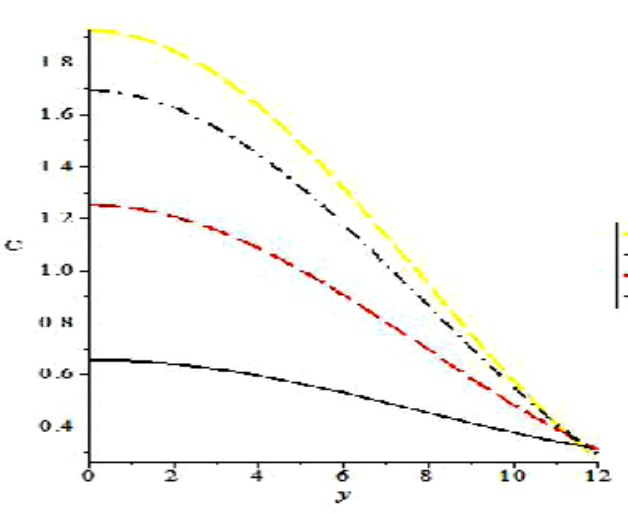
$\delta_{*}=1, \omega=0.5$.
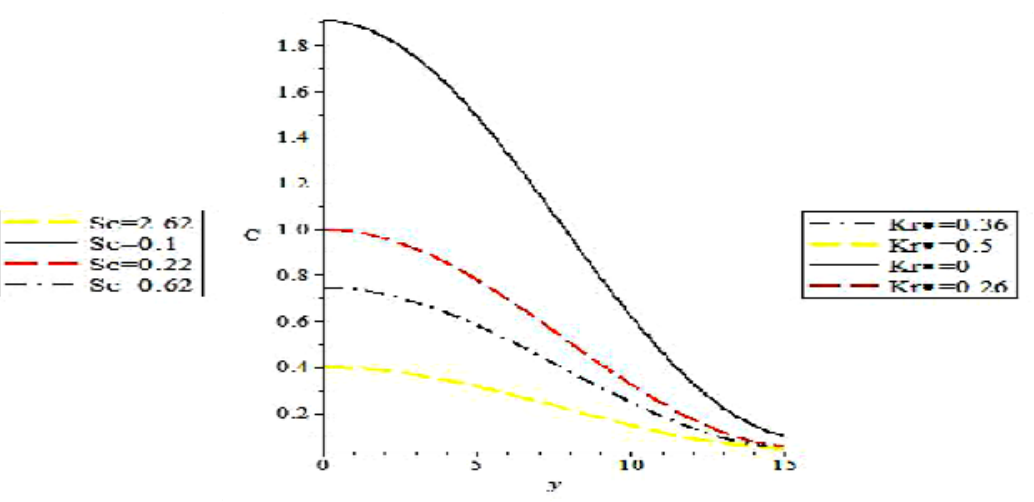

Fig.10. Effect of Sc on the Couette flow concentration Fig.11. Effect of $K r *$ on the Couette flow profile when $N_{c}=0.01, t=1.5, K r_{*}=0.05$, $\delta_{*}=1, \omega=0.2$. concentration profile when $N c=0.01, t=1.5$, $\mathrm{Sc}=0.62, \delta_{*}=1, \omega=0.2$. 

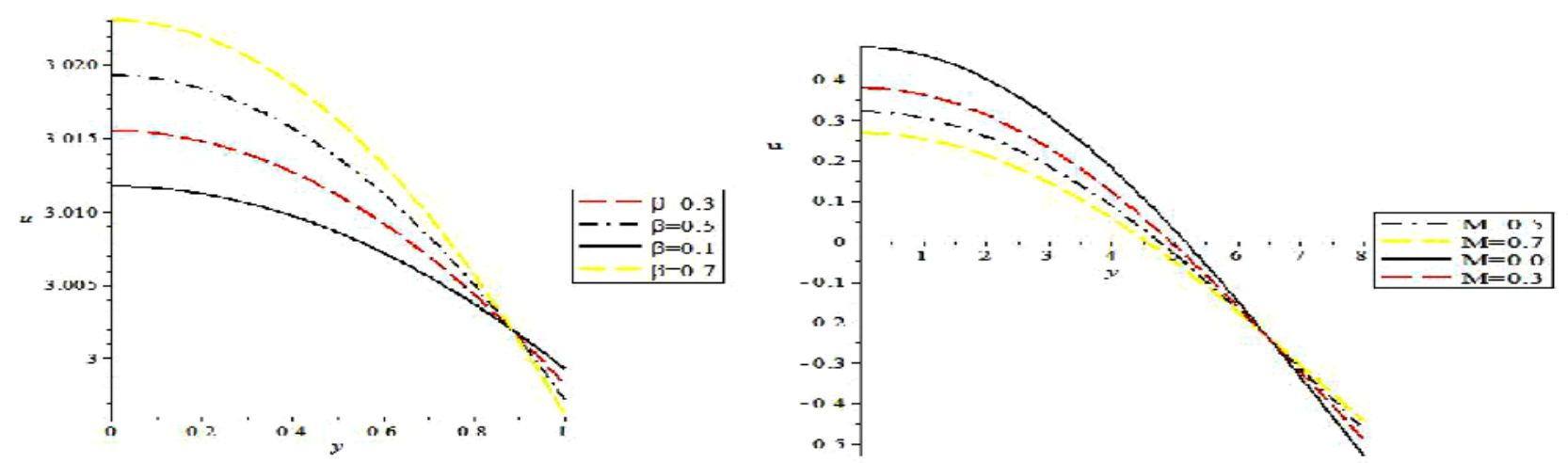

Fig.12. Effect of $\beta$ on the Poiseuille flow velocity Fig.13. Effect of $M$ on the Poiseuille flow velocity profile when $M=0.3, t=1.5, \alpha=1, \delta_{*}=1, \omega=0.2$, $\Omega=1$. profile when $\beta=1, t=1.5, \alpha=0.3, \delta_{*}=1, \omega=0.2$, $\Omega=1$.


Fig.14. Effect of $\alpha$ on the Poiseuille flow velocity Fig.15. Effect of $\Omega$ on the Poiseuille flow velocity profile when $\beta=1, M=0.3, t=0.5, \delta_{*}=1, \omega=0.2$, $\Omega=1$. profile when $\beta=0.5, \quad M=3, \quad t=1.5, \quad \alpha=0.6$,

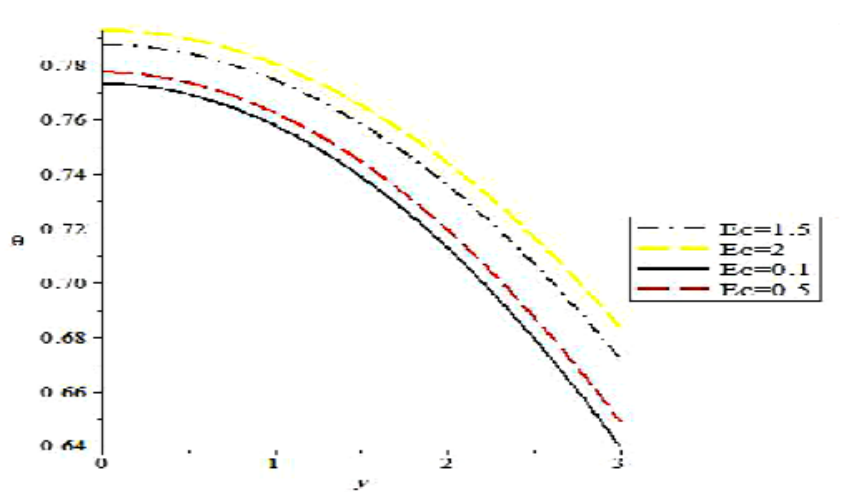
$\delta_{*}=1, \omega=0.3$.

Fig.16. Effect of $\mathrm{Ec}$ on the Poiseuille flow Fig.17. Effect of $\operatorname{Pr}$ on the Poiseuille flow temperature profile when $\beta=0.3, t=0.5, \operatorname{Pr}=0.5$, $R=1, \delta_{*}=1, \omega=0.2$.

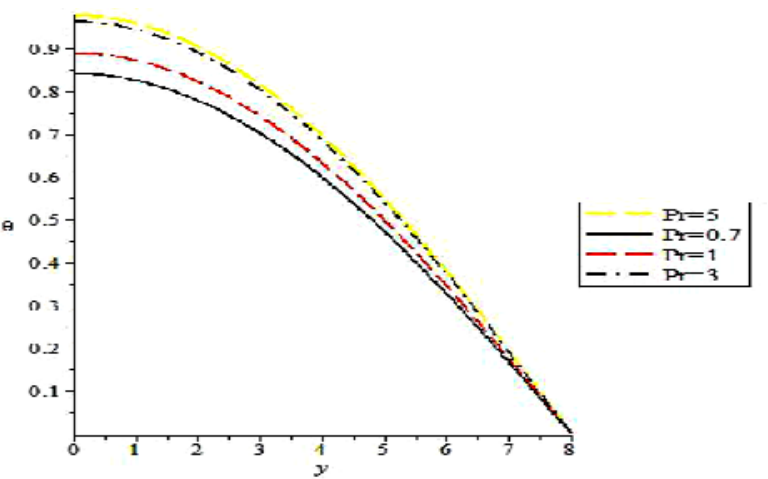
temperature profile when $\beta=0.3, \mathrm{Ec}=0.5, R=1$, $t=1.5, \delta_{*}=1, \omega=0.2$. 

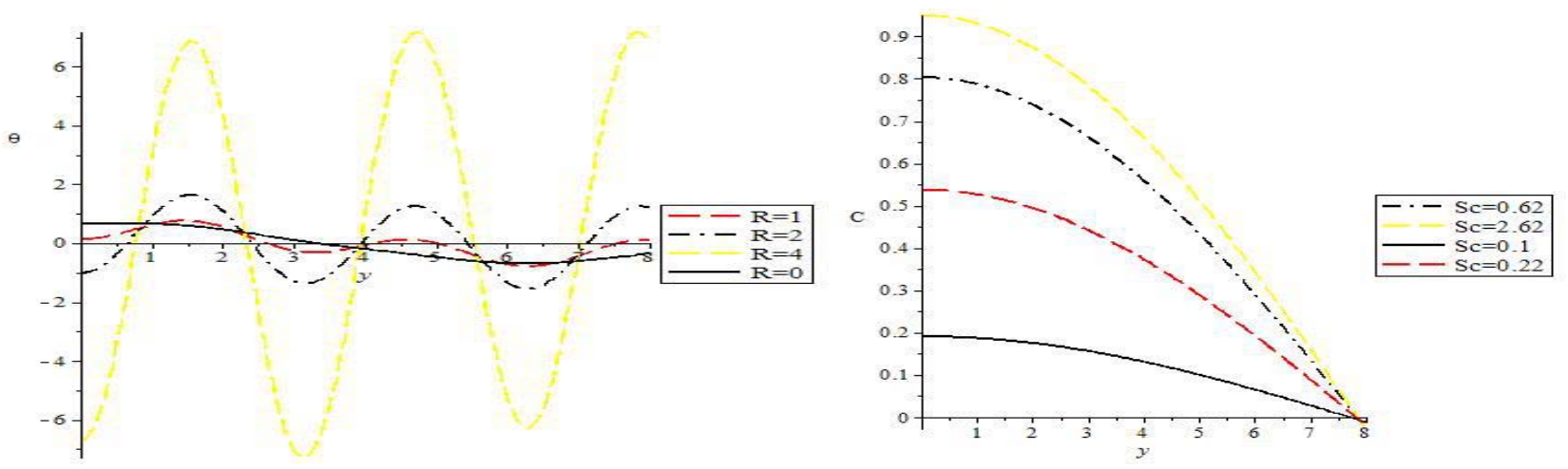

Fig.18. Effect of $R$ on the Poiseuille flow temperature Fig.19. Effect of $\mathrm{Sc}$ on the Poiseuille flow profile when $\beta=3, \quad \delta_{*}=1, \quad \omega=0.5, \quad t=1.5$, $\mathrm{Ec}=0.2, \operatorname{Pr}=1$. concentration profile when $N c=0.01, t=1.5$, $K r_{*}=0.05, \delta_{*}=1, \omega=0.2$.
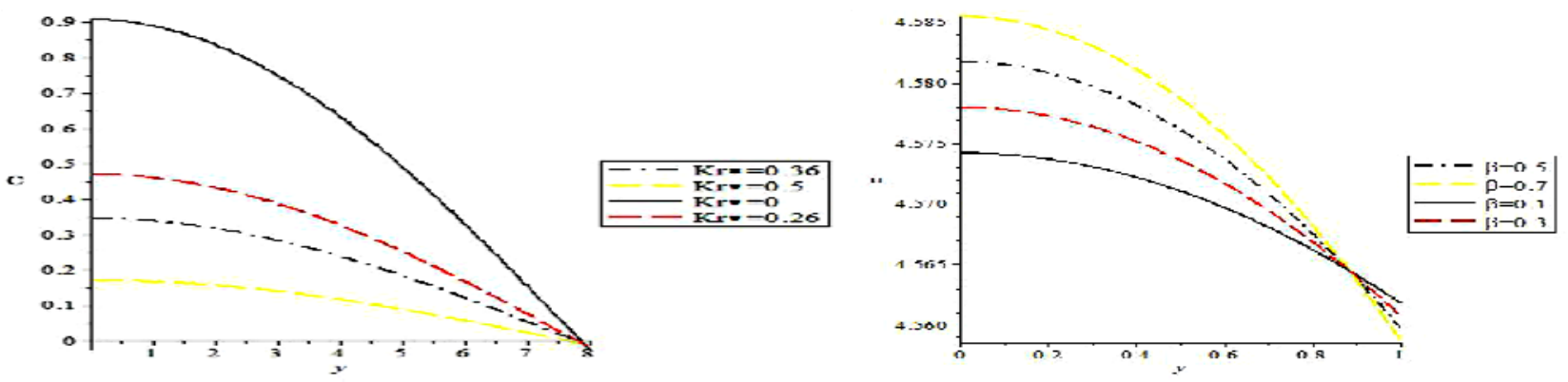

Fig.20. Effect of $K r *$ on the Poiseuille flow Fig.21. Effect of $\beta$ on the Couette-Poiseuille flow concentration profile when $N c=0.01, t=1.5$, $\mathrm{Sc}_{*}=0.62, \delta_{*}=1, \omega=0.2$. velocity profile when $M=3, \quad t=1.5, \quad \alpha=1$, $\delta_{*}=1, \omega=0.2, \Omega=1$.
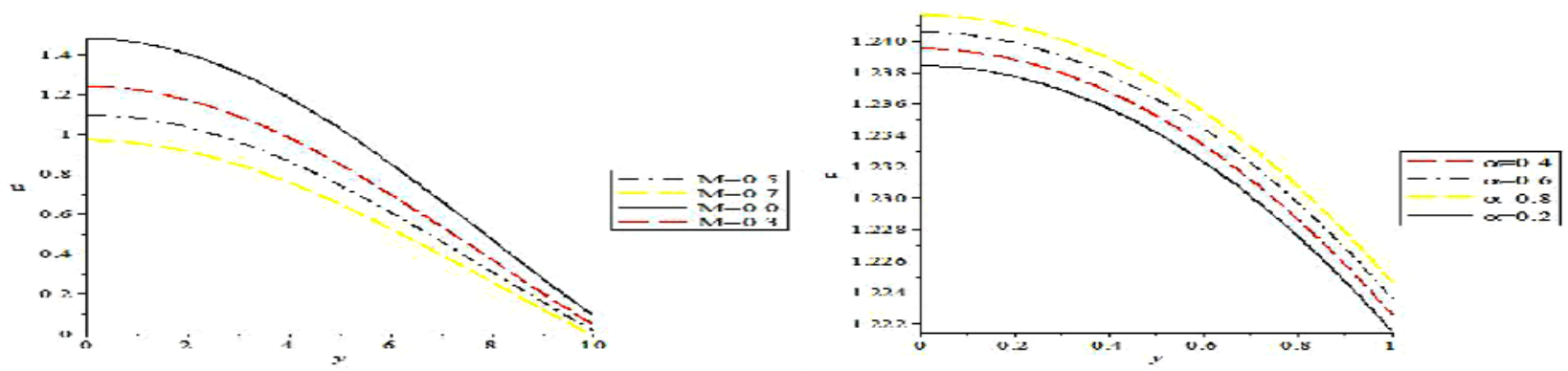

Fig.22. Effect of $M$ on the Couette-Poiseuille flow Fig.23. Effect of $\alpha$ on the Couette-Poiseuille flow velocity profile when $\beta=1, t=0.5, \alpha=0.3, \delta_{*}=1$, $\omega=0.2, \Omega=1$.

velocity profile when $\beta=1, \quad M=0.3, \quad t=0.5$, $\delta_{*}=1, \omega=0.2, \Omega=1$. 

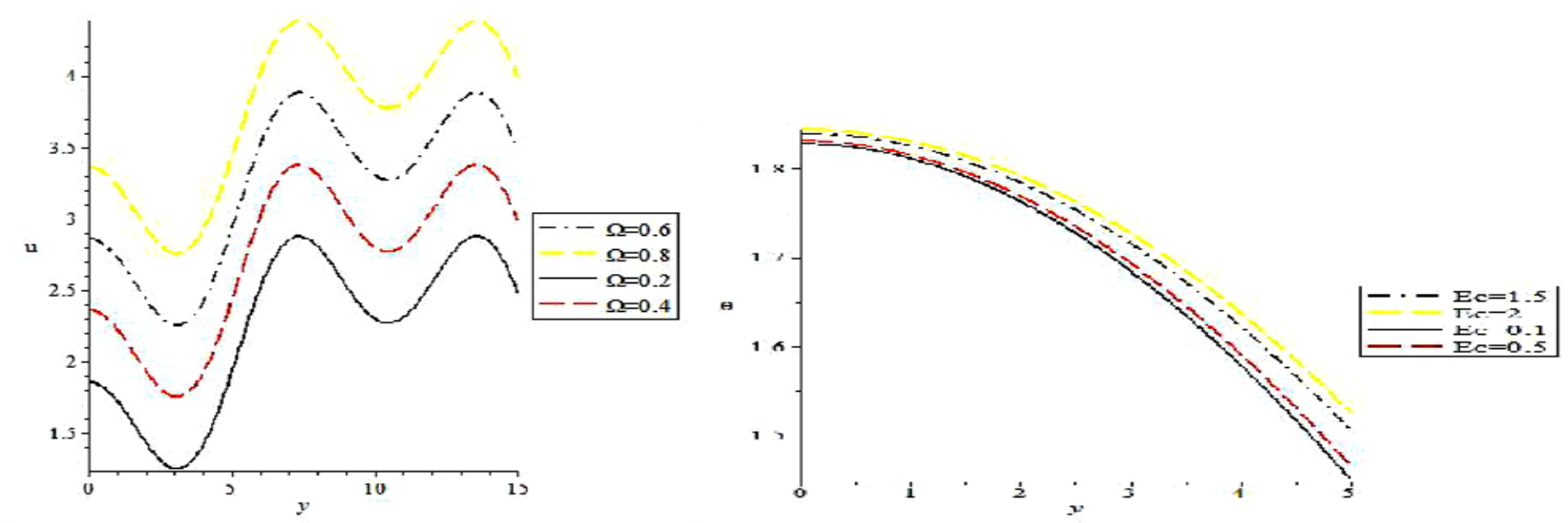

Fig.24. Effect of $\Omega$ on the Couette-Poiseuille flow Fig.25. Effect of Ec on the Couette-Poiseuille flow velocity profile when $\beta=0.5, M=0.3, t=1.5$, $\alpha=0.6, \delta_{*}=1, \omega=0.3$. temperature profile when $\beta=0.3, \operatorname{Pr}=0.5, t=1.5$, $R=0.5, \delta_{*}=1, \omega=0.2$.
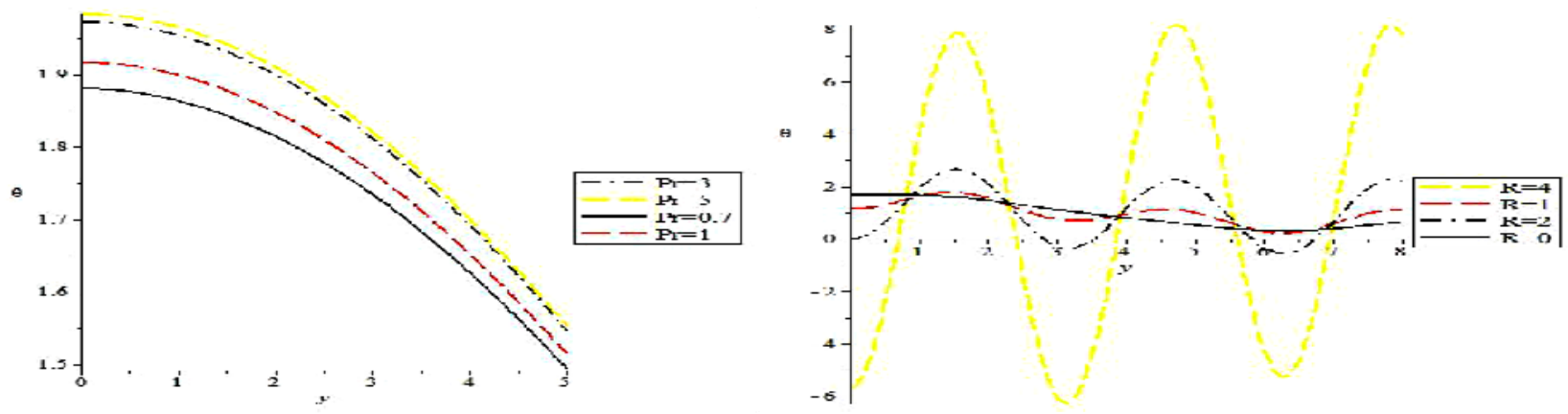

Fig.26. Effect of $\operatorname{Pr}$ on the Couette-Poiseuille flow Fig.27. Effect of $R$ on the Couette-Poiseuille flow temperature profile when $\beta=0.3, \mathrm{Ec}=0.5, t=1.5$, $R=0.5, \delta_{*}=1, \omega=0.2$. temperature profile when $\beta=3, \mathrm{Ec}=0.2, t=1.5$, $\operatorname{Pr}=1, \delta_{*}=1, \omega=0.5$.
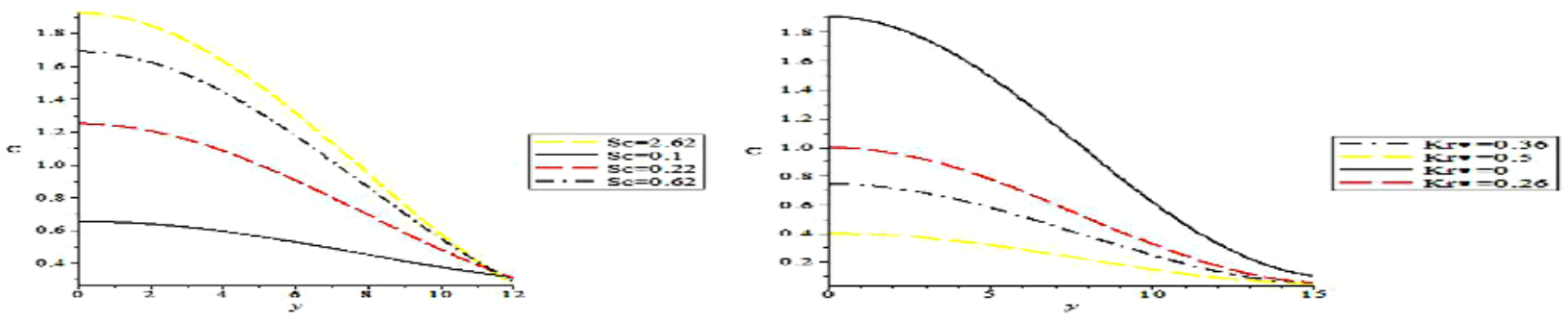

Fig.28. Effect of Sc on the Couette-Poiseuille flow Fig.29. Effect of $K r *$ on the Couette-Poiseuille flow concentration profile when $N c=0.01, t=1.5$, $K r * 0.05, \delta_{*}=1, \omega=0.2$. concentration profile when $N c=0.01, t=1.5$, $\mathrm{Sc}=0.62, \delta_{*}=1, \omega=0.2$.

Figures 4-29 show the effects of various physical parameters on the dimensionless velocity, temperature and concentration profiles for the three problems. Figures 4, 12 and 21 show the variations of velocity profiles for different values of $\beta$. It is seen from these figures that an increase in the value of $\beta$ leads to increased velocity distribution for all values of $y$. Figures 5, 13 and 22 depict the variation of velocity profiles for different values of $M$. They show that as the value $M$ increases, the velocity decreases thereby 
not reducing the viscosity of the fluid. Figures 6,14 and 23 show the velocity profiles for different values of $\alpha$. It is seen from the figures that as $\alpha$ increases the velocity profiles also increase. Figures 7, 16 and 25 show the effects of Ec on the temperature profiles. It is observed that as Ec increases, the temperature of the fluid increases with little significance and hence tends to reduce the viscosity of the fluid. Figures 8, 17 and 26 depict the variation of temperature profiles for different values of Pr. As Pr increases the temperature also increases. Figures 9, 18 and 27 show the effects of $R$ on the temperature profile. The temperature of the fluid increases as $R$ increases. Figures 10, 19 and 28 show the effects of $\mathrm{Sc}$ on the concentration profile. It is noticed that as Sc increases there is an increase in the concentration of the fluid. Figures 11, 20 and 29 present the effects of $K r_{*}$ on the concentration profile. It is found that the concentration of the fluid reduces as $K r *$ increases. Figures 15 and 24 demostrate the effects of $\Omega$ on the velocity profile. It is found that the velocity of the fluid increases with an increase in $\Omega$.

\section{Conclusion}

Effects of thermal radiation and chemical reaction on an unsteady magnetohydrodynamic(MHD) third grade fluid flow between stationary and oscillating plates are analyzed by the He-Laplace method. The solutions of velocity, temperature and concentration of the Couette flow, Poiseuille flow and CouettePoiseuille flow were presented. The following conclusions are drawn from the figures:

- velocity profiles increased due to an increase in the non-Newtonian parameter, second grade parameter and pressure gradient parameter while it decreased due to an increase in the magnetic parameter, hence increasing the boundary layer of the fluid;

- an increase in temperature profiles is a function of an increase in the Eckert number, Prandtl number and thermal radiation parameter and also reduces viscosity and boundary layer of the fluid;

- concentration profile decreased due to an increase in the chemical reaction parameter while it increased due to an increase in the Schmidt number.

\section{Nomenclature}

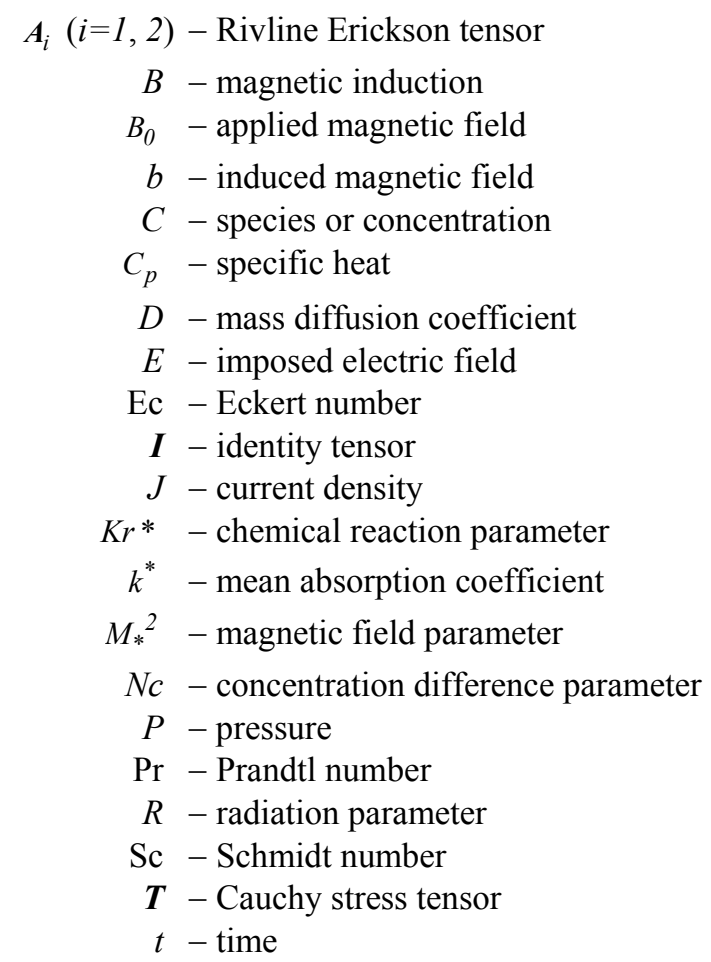




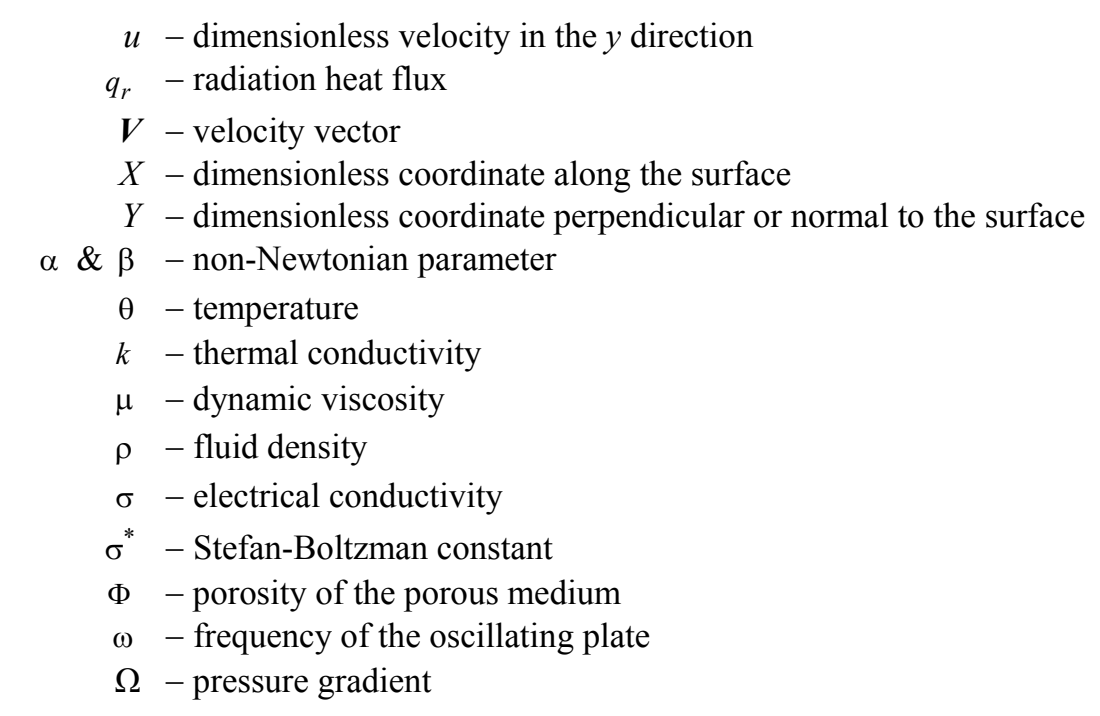

\section{REFERENCES}

[1] Hayat T., Khan M. and Ayub M. (2004): Couette and Poiseuille flow of an Oldroyd 6-constant fluid with magnetic field. - J. Math. Appl., vol.298, pp.225-244.

[2] Ajadi S.O. (2009): Analytical solutions of unsteady oscillating particulate viscoelastic fluid between two parallel walls. - International Journal of Nonlinear Science, vol.9 No2, pp.131-138.

[3] Aksoy Y. and Pakdemili M. (2010): Approximate analysis solution for flow of third grade fluid through parallel plate channel fluid with porous medium. - Transp. Porous Med, vol.83, pp.375-395.

[4] Ellahi R., Ariel P.D., Hayat T. and Asghar S. (2010): Effect of heat transfer on third grade fluid in a flat channel. Int. Journal for Numerical Methods in Fluid, vol.63, No.7, pp.847-859.

[5] Hayat T. and Nawaz M. (2010): Effect of heat transfer on magnetohydrodynamic axisymmeric flow between two stretching sheet. - Z. Naturforsch, vol.65, pp.961-968.

[6] Danish M., Kumar S. and Kumar S. (2012): Exact analytical solutions for the Poiseuille and Couette-Poiseuille flow of third grade fluid between parallel plates. - Comm. in Nonlinear Science and Numerical Simulation, vol.17, No.3, pp.1089-1097.

[7] Gbadeyan J.A. and Dada M.S. (2013): On the influence of radiation and heat transfer on an unsteady MHD nonNewtonian fluid flow with slip in a porous medium. - Journal of Mathematical Research, vol.5, No.3, pp.40-50.

[8] Aiyesimi Y.M., Okedayo G.T. and Lawal O.W. (2014a): Analysis of unsteady MHD thin film flow of a third grade fluid with heat transfer down an inclined plate. - J. Appl. Computat. Math, vol.3, No.2, pp.1-12, dio:10.4172/2168-9679.1000152.

[9] Aiyesimi Y.M., Okedayo G.T. and Lawal O.W. (2014b): Effects of magnetic field on the MHD flow of a third grade fluid through inclined channel with ohmic heating. - J. Appl. Computat. Math, vol.3, No.2, pp.1-6, dio:10.4172/2168-9679.1000153.

[10] Baoku I.G. (2014): Effects of suction and thermal radiation on heat transfer in a third grade fluid over a vertical plate. - Physical Science International Journal, vol.4, No.9, pp.1293-1310.

[11] Gul T., Islam S., Shah R.A., Khan I. and Shafie S. (2014): Thin film flow in MHD third grade fluid on a vertical belt with temperature dependent viscosity. - PLOS ONE, vol.9, No.6, e97552.

[12] Hayat T., Shafiq A. and Alsaedi A. (2014): Effect of Joule heating and thermal radiation in flow of third grade fluid over radiative surface. - PLOS ONE, vol.9, No.1, e83153.

[13] Rasheed H., Gul T., Islam S., Nasir S., Khan M.A. and Gul A. (2014): Study of Couette and Poiseuille flows of an unsteady MHD third grade fluid. - J. Appl. Environ. Biol. Sci.,vol.4, No.10, pp.12-21. 
[14] Shah S.A., Gul T., Islam S., Nasir S., Khan M.A. and Shah R.A. (2014): HPM solution of an unsteady nonNewtonian fluid between stationary and oscilating plates. - J. Appl. Environ. Biol. Sci., vol.4, No.12, pp.203-212.

[15] Hayat T., Shafiq A. and Alsaedi A. and Asghar S. (2015): Effect of inclined magnetic field in flow of third grade fluid with variable thermal conductivity. - AIP Advances, vol.5, 087108(1-15).

[16] Makinde O.D., Egunjobi A.S. and Tshehla M.S. (2015): Thermodynamics analysis of variable viscosity hydromagnetic Couette flow in a rotating system with hall effects. - Entropy, vol.17, pp.7811-7826.

[17] Nasir S., Gul T., Islam S., Shah R.A. (2015): Unsteady MHD flow and heat transfer of third grade fluid past on oscillating inclined belt. - Science Postprint, vol.1, No.2, 200045.

[18] Ghani F., Gul T., Islam S., Shah R.A., Khan I., Sharida S., Nasir S. and Khan M.A. (2016): Unsteady magnetohydrodynamics thin film flow of a third grade fluid over an oscillating inclined belt embedded in a porous medium. - Thermal Science, vol.5, pp.875-887.

[19] Kumar M.S., Sandeep N. and Kumar B.R. (2016): Effect of nonlinear thermal radiation on unsteady MHD flow between parallel plate. - Global Journal of Pure and Applied Mathematics, vol.2, No.1, pp.0973-1768.

[20] Hradyesh K.M. and Atulya K.N. (2012): He-Laplace method for linear and nonlinear partial differential equations. - Journal of Applied Mathematics, pp.1-16.

[21] Ghorbani A. and Saberi-Nadjafi J. (2007): He's homotopy perturbation method for calculating Adomian polynomial. - International Journal of Nonlinear Science and Numerical Simulation, vol.8, pp.229-232.

[22] Ghorbani A. (2009): Beyond Adomian polynomials: He polynomials. - Chaos Solitons and Fractals, vol.39, No.3, pp.1486-1492.

Received: May 12, 2018

Revised: November 18, 2018 